\title{
Channel Equalization in Filter Bank Based Multicarrier Modulation for Wireless Communications
}

\author{
Tero Ihalainen, ${ }^{1}$ Tobias Hidalgo Stitz, ${ }^{1}$ Mika Rinne, ${ }^{2}$ and Markku Renfors ${ }^{1}$ \\ ${ }^{1}$ Institute of Communications Engineering, Tampere University of Technology, P.O. Box 553, Tampere FI-33101, Finland \\ ${ }^{2}$ Nokia Research Center, P.O. Box 407, Helsinki FI-00045, Finland
}

Received 5 January 2006; Revised 6 August 2006; Accepted 13 August 2006

Recommended by See-May Phoong

\begin{abstract}
Channel equalization in filter bank based multicarrier (FBMC) modulation is addressed. We utilize an efficient oversampled filter bank concept with $2 \mathrm{x}$-oversampled subcarrier signals that can be equalized independently of each other. Due to Nyquist pulse shaping, consecutive symbol waveforms overlap in time, which calls for special means for equalization. Two alternative linear low-complexity subcarrier equalizer structures are developed together with straightforward channel estimation-based methods to calculate the equalizer coefficients using pointwise equalization within each subband (in a frequency-sampled manner). A novel structure, consisting of a linear-phase FIR amplitude equalizer and an allpass filter as phase equalizer, is found to provide enhanced robustness to timing estimation errors. This allows the receiver to be operated without time synchronization before the filter bank. The coded error-rate performance of FBMC with the studied equalization scheme is compared to a cyclic prefix OFDM reference in wireless mobile channel conditions, taking into account issues like spectral regrowth with practical nonlinear transmitters and sensitivity to frequency offsets. It is further emphasized that FBMC provides flexible means for high-quality frequency selective filtering in the receiver to suppress strong interfering spectral components within or close to the used frequency band.
\end{abstract}

Copyright (c) 2007 Tero Ihalainen et al. This is an open access article distributed under the Creative Commons Attribution License, which permits unrestricted use, distribution, and reproduction in any medium, provided the original work is properly cited.

\section{INTRODUCTION}

Orthogonal frequency division multiplexing (OFDM) [1] has become a widely accepted technique for the realization of broadband air-interfaces in high data rate wireless access systems. Indeed, due to its inherent robustness to multipath propagation, OFDM has become the modulation choice for both wireless local area network (WLAN) and terrestrial digital broadcasting (digital audio and video broadcasting; DAB, DVB) standards. Furthermore, multicarrier transmission schemes are generally considered candidates for the future "beyond 3 G" mobile communications.

All these current multicarrier systems are based on the conventional cyclic prefix OFDM modulation scheme. In such systems, very simple equalization (one complex coefficient per subcarrier) is made possible by converting the broadband frequency selective channel into a set of parallel flat-fading subchannels. This is achieved using the inverse fast Fourier transform (IFFT) processing and by inserting a time domain guard interval, in the form of a cyclic prefix (CP), to the OFDM symbols at the transmitter. By dimensioning the $\mathrm{CP}$ longer than the maximum delay spread of the radio channel, interference from the previous OFDM symbol, referred to as inter-symbol-interference (ISI), will only affect the guard interval. At the receiver, the guard interval is discarded to elegantly avoid ISI prior to transforming the signal back to frequency domain using the fast Fourier transform (FFT).

While enabling a very efficient and simple way to combat multipath effects, the $\mathrm{CP}$ is pure redundancy, which decreases the spectral efficiency. As a consequence, there has recently been a growing interest towards alternative multicarrier schemes, which could provide the same robustness without requiring a $\mathrm{CP}$, that is, offering improved spectral efficiency. Pulse shaping in multicarrier transmission dates back to the early work of Chang [2] and Saltzberg [3] in the sixties. Since then, various multicarrier concepts based on the Nyquist pulse shaping idea with overlapping symbols and bandlimited subcarrier signals have been developed by Hirosaki [4], Le Floch et al. [5], Sandberg and Tzannes [6], Vahlin and Holte [7], Wiegand and Fliege [8], Nedic [9], Vandendorpe et al. [10], Van Acker et al. [11], Siohan et al. [12], Wyglinski et al. [13], Farhang-Boroujeny [14, 15], Phoong et al. [16], and others. One central ingredient in the 
later developments is the theory of efficiently implementable, modulation-based uniform filter banks, developed by Vetterli [17], Malvar [18], Vaidyanathan [19], and Karp and Fliege [20], among others. In this context, the filter banks are used in a transmultiplexer (TMUX) configuration.

We refer to the general concept as filter bank based multicarrier (FBMC) modulation. In FBMC, the subcarrier signals cannot be assumed flat-fading unless the number of subcarriers is very high. One approach to deal with the fading frequency selective channel is to use waveforms that are well localized, that is, the pulse energy both in time and frequency domains is well contained to limit the effect on consecutive symbols and neighboring subchannels $[5,7,12]$. In this context, a basic subcarrier equalizer structure of a single complex coefficient per subcarrier is usually considered. Another approach uses finite impulse response (FIR) filters as subcarrier equalizers with cross-connections between the adjacent subchannels to cancel the inter-carrier-interference (ICI) $[6,10]$. A third line of studies applies a receiver filter bank structure providing oversampled subcarrier signals and performs persubcarrier equalization using FIR filters $[4,8,9,11,13]$. The main idea here is that equalization of the oversampled subcarrier signals restores the orthogonality of the subcarrier waveforms and there is no need for cross-connections between the subcarriers. This paper contributes to this line of studies by developing low-complexity linear per-subcarrier channel equalizer structures for FBMC. The earlier contributions either lack connection to the theory of efficient multirate filter banks, use just a complex multiplier as subcarrier equalizer or, in case of non trivial subcarrier equalizers, lack the analysis of needed equalizer length in practical wireless communication applications (many of such studies have focused purely on wireline transmission). Also various practical issues like peak-to-average power ratio and effects of timing and frequency offsets have not properly been addressed in this context before.

The basic model of the studied adaptive sine modulated/cosine modulated filter bank equalizer for transmultiplexers (ASCET) has been presented in our earlier work [21-23]. This paper extends the low-complexity equalizer of $[23,24]$, presenting comprehensive performance analysis, and studies the tradeoffs between equalizer complexity and number of subcarriers required to achieve close-to-ideal performance in a practical broadband wireless communication environment. A simple channel estimation-based calculation of the equalizer coefficients is presented. The performance of the studied equalizer structures is compared to OFDM, taking into account various practical issues.

In a companion paper [25], a similar subband equalizer structure is applied to the filter bank approach for frequency domain equalization in single carrier transmission. In that context, filter banks are used in the analysis-synthesis configuration to replace the traditional FFT-IFFT transform-pair in the receiver.

The rest of the paper is organized as follows. Section 2 briefly describes an efficient implementation structure for the TMUX based on exponentially modulated filter banks (EMFB) [26]. The structure consists of a critically sampled synthesis and a 2x-oversampled analysis bank. The problem of channel equalization is addressed in Section 3. The theoretical background and principles of the proposed compensation method are presented. The chosen filter bank structure leads to a relatively simple signal model that results in criteria for perfect subcarrier equalization and formulas for FBMC performance analysis in case of practical equalizers. A complex FIR filter-based subcarrier equalizer (CFIR-SCE) and the so-called amplitude-phase (AP-SCE) equalizer are presented. Especially, some low-complexity cases are analyzed and compared in Section 4. In Section 5, we present a semianalytical and a full time domain simulation setup to evaluate the performance of the equalizer structures in a broadband wireless communication channel. Furthermore, the effects of timing and frequency offsets, nonlinearity of a power amplifier, and overall system complexity are briefly investigated. Finally, the conclusions are drawn in Section 6.

\section{EXPONENTIALLY MODULATED PERFECT RECONSTRUCTION TRANSMULTIPLEXER}

Figure 1 shows the structure of the complex exponentially modulated TMUX that can produce a complex inphase/quadrature (I/Q) baseband signal required for spectrally efficient radio communications [23]. It has real format for the low-rate input signals and complex I/Q-presentation for the high-rate channel signal. It should be noted that FBMC with (real) $m$-PAM as subcarrier modulation and OFDM with (complex) $m^{2}$-QAM ideally provide the same bit rate since in general the subcarrier symbol rate in FBMC is twice that of OFDM for a fixed subchannel spacing. In this structure, there are $2 M$ low-rate subchannels equally spaced between $\left[-F_{s} / 2, F_{s} / 2\right], F_{s}$ denoting the high sampling rate.

EMFBs belong to a class of filter banks in which the subfilters are formed by frequency shifting the lowpass prototype $h_{p}[n]$ with an exponential sequence [27]. Exponential modulation translates $H_{p}\left(e^{j \omega}\right)$ (lowpass frequency response) around the new center frequency determined by the subcarrier index $k$. The prototype $h_{p}[n]$ can be optimized in such a manner that the filter bank satisfies the perfectreconstruction $(\mathrm{PR})$ condition, that is, the output signal is a delayed version of the input signal $[27,28]$. In the general form, the synthesis and analysis filters of EMFBs can be written as

$$
\begin{aligned}
& f_{k}[n]=\sqrt{\frac{2}{M}} h_{p}[n] \exp \left(j\left[n+\frac{M+1}{2}\right]\left[k+\frac{1}{2}\right] \frac{\pi}{M}\right), \\
& h_{k}[n]=\sqrt{\frac{2}{M}} h_{p}[n] \exp \left(-j\left[N-n+\frac{M+1}{2}\right]\left[k+\frac{1}{2}\right] \frac{\pi}{M}\right),
\end{aligned}
$$

respectively, where $n=0,1, \ldots, N$ and $k=0,1, \ldots, 2 M-1$. Furthermore, it is assumed that the filter order is $N=2 \mathrm{KM}-$ 1 . The overlapping factor $K$ can be used as a design parameter because it affects on how much stopband attenuation can be achieved. Another essential design parameter is the stopband edge of the prototype filter $\omega_{s}=(1+\rho) \pi / 2 M$, where 


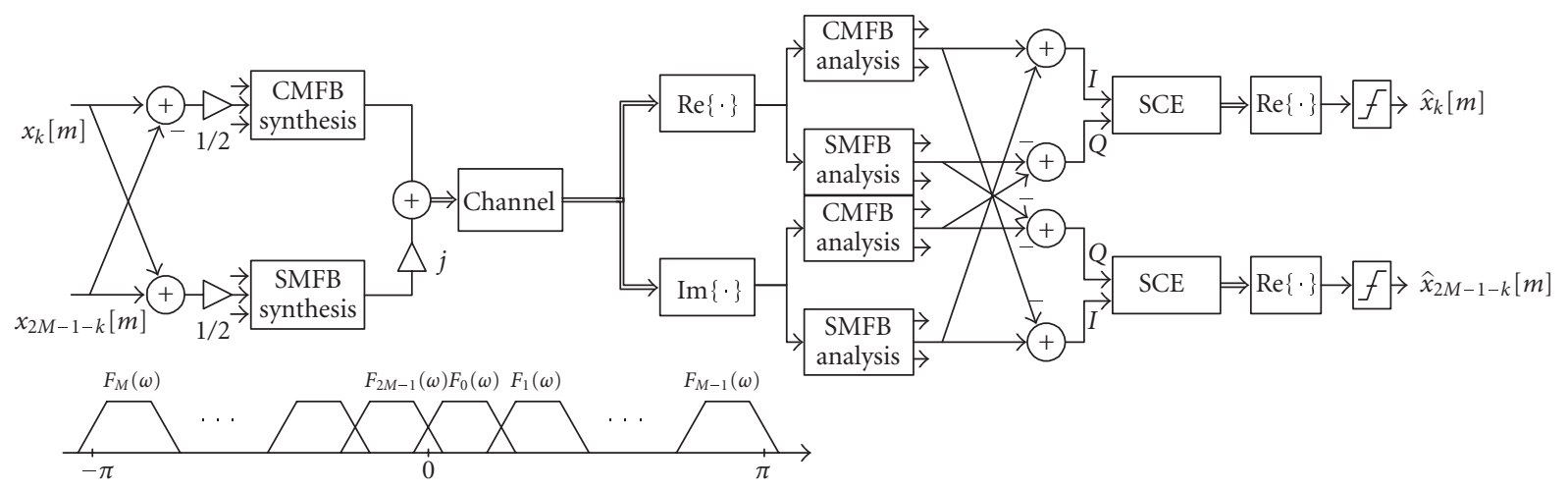

Figure 1: Complex TMUX with oversampled analysis bank and per-subcarrier equalizers.

the roll-off parameter $\rho$ determines how much adjacent subchannels overlap. Typically, $\rho=1.0$ is used, in which case only the contiguous subchannels are overlapping with each other, and the overall subchannel bandwidth is twice the subchannel spacing.

In the approach selected here, the EMFB is implemented using cosine and sine modulated filter bank (CMFB/SMFB) blocks [28], as can be seen in Figure 1. The extended lapped transform (ELT) is an efficient method for implementing PR CMFBs [18] and SMFBs [28]. The relations between the synthesis and analysis filters of the $2 M$-channel EMFB and the corresponding $M$-channel CMFB and SMFB with the same real FIR prototype $h_{p}[n]$ are

$$
\begin{gathered}
f_{k}[n]= \begin{cases}f_{k}^{c}[n]+j f_{k}^{s}[n], & k \in[0, M-1] \\
-\left(f_{2 M-1-k}^{c}[n]-j f_{2 M-1-k}^{s}[n]\right), & k \in[M, 2 M-1],\end{cases} \\
h_{k}[n]= \begin{cases}h_{k}^{c}[n]-j h_{k}^{s}[n], & k \in[0, M-1] \\
-\left(h_{2 M-1-k}^{c}[n]+j h_{2 M-1-k}^{s}[n]\right), & k \in[M, 2 M-1],\end{cases}
\end{gathered}
$$

respectively. A specific feature of the structure in Figure 1 is that while the synthesis filter bank is critically sampled, the subchannel output signals of the analysis bank are oversampled [26] by a factor of two. This is achieved by using the symbol-rate complex (I/Q) subchannel signals, instead of the real ones that are sufficient for detection after the channel equalizer, or in case of a distortion-free channel.

We consider here the use of EMFBs which have odd channel stacking, that is, the center-most pair of subchannels is symmetrically located around the zero frequency at the baseband. We could equally well use a modified EMFB structure [26] with even stacking (the center-most subchannel located symmetrically about zero). The latter form has also a slightly more efficient implementation structure, based on DFT-processing. The proposed equalizer structure can also be applied with modified DFT (MDFT) filter banks [20], with modified subchannel processing. However, for the following analysis EMFB was selected since it results in the most straightforward system model.
Further, although the discussion here is based on the use of PR filter banks, also nearly perfect-reconstruction (NPR) designs could be utilized. In the critically sampled case, the implementation benefits of NPR designs are limited because the efficient ELT structures cannot be utilized [29]. However, in the 2x-oversampled case, having two parallel CMFB and SMFB blocks, the implementation benefits of NPR designs could be more significant.

\section{CHANNEL EQUALIZATION}

The problem of channel equalization in the FBMC context is not so well understood as in the DFT-based systems. Our equalizer concept can be applied to both real and complex modulated baseband signal formats; here we focus on the complex case. In its simplest form, the subcarrier equalizer structure consists only of a single complex coefficient that adjusts the amplitude and phase responses of each subchannel in the receiver [22]. Higher-order SCEs are able to equalize each subchannel better if the channel frequency response is not flat within the subchannel. As a result, the use of higher-order SCEs enables to increase the relative subchannel bandwidth because the subchannel responses are allowed to take mildly frequency selective shapes. As a consequence, the number of subchannels to cover a given signal bandwidth by FBMC can be reduced. In general, higher-order equalizer structures provide flexibility and scalability to system design because they offer a tradeoff between the number of required subchannels and complexity of the subcarrier equalizers.

The oversampled receiver is essential for the proposed equalizer structure. In case of roll-off $\rho=1.0$ or lower, nonaliased versions of the subchannel signals are obtained in the $2 \mathrm{x}$-oversampled receiver when complex (I/Q) signals are sampled at the symbol rate. Consequently, complete channel equalization in an optimal manner is possible. As a result of the high stopband attenuation of the subchannel filters, there is practically no aliasing of the subchannel signals in the receiver bank. Thus perfect equalization of the distorting channel within the subchannel passband and transition band regions would completely restore the orthogonality of the subchannel signals [9]. 


\subsection{Theoretical background and principles}

Figure 2(a) shows a subchannel model of the complex TMUX with per-subcarrier equalizer. A more detailed model that includes the interference from the contiguous subchannels is shown in Figure 2(b). Limiting the sources of interference to the closest neighboring subchannels is justified if the filter bank design provides sufficiently high stopband attenuation. Furthermore, in this model the order of downsampling and equalization is interchanged based on the multirate identities [19]. The latter model is used as a basis for the cross-talk analysis that follows. It is also convenient for semianalytical performance evaluations. The equalizer concept is based on the property that with ideal sampling and equalization, the desired subchannel signal, carried by the real part of the complex subchannel output, is orthogonal to the contiguous subchannel signal components occupying the imaginary part. The orthogonality between the subchannels is introduced when the linear-phase lowpass prototype $h_{p}[n]$ is exponentially frequency shifted as a bandpass filter, with 90-degree phase-shift between the carriers of the contiguous subchannels.

In practice, the nonideal channel causes amplitude and phase distortion. The latter results in rotation between the I-and Q-components of the neighboring subchannel signals causing ICI or cross-talk between the subchannels. ISI, on the other hand, is mainly caused by the amplitude distortion. The following set of equations provides proofs for these statements. We derive them for an arbitrary subchannel $k$ on the positive side of the baseband spectrum and the results can easily be extended for the subchannels on the negative side using (3) and (4). In the following analysis we use a noncausal zero-phase system model, which is obtained by using, instead of (2), analysis filters of the form

$$
\bar{h}_{k}[n]=\sqrt{\frac{2}{M}} h_{p}[n+N] \exp \left(-j\left[-n+\frac{M+1}{2}\right]\left[k+\frac{1}{2}\right] \frac{\pi}{M}\right) .
$$

By referring to the equivalent form, shown in Figure 2(b), and adopting the notation from there, we can express the cascade of the synthesis and analysis filters of the desired subchannel $k$ as

$$
\begin{aligned}
f_{k}[n] * \bar{h}_{k}[n]= & \sum_{l=a}^{b} \bar{h}_{k}^{c}[l] f_{k}^{c}[n-l]+\sum_{l=a}^{b} \bar{h}_{k}^{s}[l] f_{k}^{s}[n-l] \\
& +j \cdot\left(\sum_{l=a}^{b} \bar{h}_{k}^{c}[l] f_{k}^{s}[n-l]-\sum_{l=a}^{b} \bar{h}_{k}^{s}[l] f_{k}^{c}[n-l]\right) \\
= & t_{k}^{I}[n]+j \cdot t_{k}^{Q}[n]=t_{k}[n],
\end{aligned}
$$

where $*$ denotes the convolution operation, summation indexes are $a=-N+\max (n, 0)$ and $b=\min (n, 0)$, and $n \in[-N, \ldots, N]$.

\subsubsection{IClanalysis}

For the potential ICI terms from the contiguous subchannels $k-1$ and $k+1$ (below and above) to the subchannel $k$ of interest, we can write

$$
\begin{aligned}
f_{k-1}[n] & * \bar{h}_{k}[n] \\
= & \sum_{l=a}^{b} \bar{h}_{k}^{c}[l] f_{k-1}^{c}[n-l]+\sum_{l=a}^{b} \bar{h}_{k}^{s}[l] f_{k-1}^{s}[n-l] \\
& +j \cdot\left(\sum_{l=a}^{b} \bar{h}_{k}^{c}[l] f_{k-1}^{s}[n-l]-\sum_{l=a}^{b} \bar{h}_{k}^{s}[l] f_{k-1}^{c}[n-l]\right) \\
= & v_{k}^{I}[n]+j \cdot v_{k}^{Q}[n]=v_{k}[n], \\
f_{k+1}[n] & * \bar{h}_{k}[n] \\
= & \sum_{l=a}^{b} \bar{h}_{k}^{c}[l] f_{k+1}^{c}[n-l]+\sum_{l=a}^{b} \bar{h}_{k}^{s}[l] f_{k+1}^{s}[n-l] \\
& +j \cdot\left(\sum_{l=a}^{b} \bar{h}_{k}^{c}[l] f_{k+1}^{s}[n-l]-\sum_{l=a}^{b} \bar{h}_{k}^{s}[l] f_{k+1}^{c}[n-l]\right) \\
= & u_{k}^{I}[n]+j \cdot u_{k}^{Q}[n]=u_{k}[n],
\end{aligned}
$$

respectively.

Due to PR design, the real parts $v_{k}^{I}[m]$ and $u_{k}^{I}[m]$ ( $m$ being the sample index at the low rate) of the downsampled subchannel signals are all-zero sequences (or close to zero sequences in the NPR case). So ideally, when the real part of the signal is taken in the receiver, no crosstalk from the neighboring subchannels is present in the signal used for detection. Channel distortion, however, causes phase rotation between the I- and Q-components breaking the orthogonality between the subcarriers. Channel equalization is required to recover the orthogonality of the subcarriers.

The ICI components from other subcarriers located further apart from the subchannel of interest are considered negligible. This is a reasonable assumption because the extent of overlapping of subchannel spectra and the level of stopband attenuation can easily be controlled in FBMC. In fact, they are used as optimization criteria in filter bank design, as discussed in the previous section.

The cascade of the distorting channel with instantaneous impulse response (in the baseband model) $h_{\mathrm{ch}}[n]$ and the upsampled version of the per-subcarrier equalizer $\bar{c}_{k}[n]$ (see Figure 2) applied to the subchannel $k$ of interest can be expressed as

$$
h_{\mathrm{ch}}[n] * \bar{c}_{k}[n]=r_{k}[n] .
$$

In the analysis, a noncausal high-rate impulse response $\bar{c}_{k}[n]$ is used for the equalizer, although in practice the low-rate causal form $c_{k}[m]$ is applied.

Next we analyze the ICI components potentially remaining in the real parts of the subchannel signals that are used for detection. Figure 3 visualizes the two ICI bands for subchannel $k=0$. We start from the lower-side ICI term and use an equivalent baseband model, where the potential ICI energy 


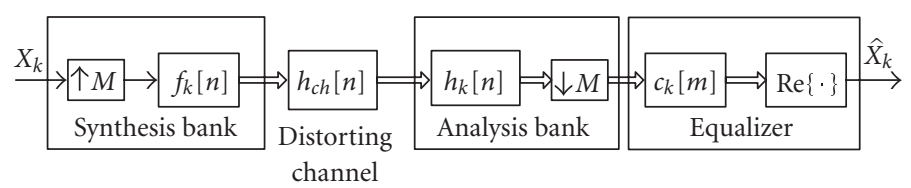

(a)

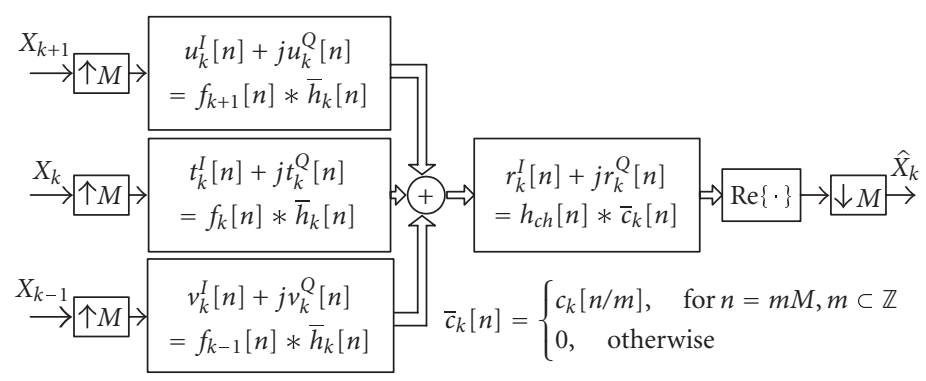

(b)

FIGURE 2: Complex TMUX with per-subcarrier equalizer. (a) System model for subchannel $k$. (b) Equivalent form including also contiguous subchannels for crosstalk analysis.

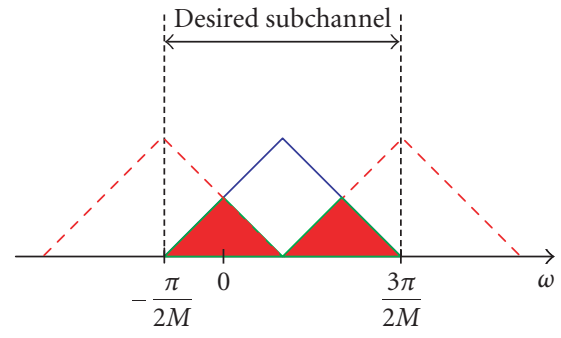

_ RX filter of the desired subchannel

- - - TX filter of the contiguous subchannel

Potential ICI spectrum

FIgURE 3: Potential ICI spectrum for subchannel $k=0$.

is symmetrically located about zero frequency. We can write the baseband cross-talk impulse response from subchannel $k-1$ to subchannel $k$ in case of an ideal channel as

$$
\tilde{v}_{k}[n]=\widetilde{v}_{k}^{I}[n]+j \tilde{v}_{k}^{Q}[n]=v_{k}[n] e^{-j n k \pi / M} .
$$

In the appendix, it is shown that this impulse response is purely imaginary, that is, $\widetilde{v}_{k}^{I}[n] \equiv 0$ and $\widetilde{v}_{k}[n]=v_{0}[n]$. In case of nonideal channel with channel equalization, the baseband cross-talk impulse response can now be written as

$$
\tilde{g}_{k}^{k-1}[n]=j v_{0}^{Q}[n] * \widetilde{r}_{k}[n],
$$

where $\tilde{r}_{k}[n]=r_{k}[n] e^{-j n k \pi / M}$. Here the upper index denotes the source of ICI. Now we can see that if the equalized channel impulse response is real in the baseband model, then the cross-talk impulse response is purely imaginary, and there is no lower-side ICI in the real part of the subchannel signal that is used for detection.

At this point we have to notice that the lower-side ICI energy is zero-centered after decimation only for the evenindexed subchannels, and for the odd subchannels the above model is not valid as such. However, we can establish a simple relation between the actual decimated subchannel output sequence $z_{k}[m M]$ in the filter bank system and the sequence obtained by decimating in the baseband model. It is straightforward to see that the following relation holds:

$$
\left.z_{k}[n] e^{-j n k \pi / M}\right|_{n=m M}=(-1)^{m k} z_{k}[m M] .
$$

Thus, for odd subchannels, the actual decimated ICI sequence is obtained by lowpass-to-highpass transformation (i.e., through multiplication by an alternating \pm 1 -sequence) from the ICI sequence of the baseband model. Then the actual ICI is guaranteed to be zero if it is zero in the baseband model. Therefore, a sufficient condition for zero lower-side ICI in all subchannels is that the equalized baseband channel impulse response is purely real.

For the upper-side ICI, we can first write the baseband model as

$$
\tilde{u}_{k}[n]=\tilde{u}_{k}^{I}[n]+j \tilde{u}_{k}^{Q}[n]=u_{k}[n] e^{-j n(k+1) \pi / M} .
$$

Again, it is shown in the appendix that this baseband impulse response is purely imaginary, that is, $\tilde{u}_{k}^{I}[n] \equiv 0$ and $\tilde{u}_{k}[n]=u_{2 M-1}[n]$. With equalized nonideal channel, the cross-talk response is now

$$
\tilde{g}_{k}^{k+1}[n]=j u_{2 M-1}^{Q}[n] *\left(\tilde{r}_{k}[n] e^{-j n \pi / M}\right)
$$

and the upper-side ICI vanishes if the equalized channel impulse response is real in this baseband model. Now the relation between the decimated models is

$$
\left.z_{k}[n] e^{-j n(k+1) \pi / M}\right|_{n=m M}=(-1)^{m(k+1)} z_{k}[m M]
$$


and a sufficient condition also for zero upper-side ICI is that the equalized baseband channel impulse response is purely real. However, the baseband models for the two cases are slightly different, and both conditions

$$
\begin{gathered}
\operatorname{Im}\left[\tilde{r}_{k}[n]\right] \equiv 0, \\
\operatorname{Im}\left[\tilde{r}_{k}[n] e^{-j n \pi / M}\right] \equiv 0
\end{gathered}
$$

have to be simultaneously satisfied to achieve zero overall ICI. In frequency domain, the equalized channel frequency response is required to have symmetric amplitude and antisymmetric phase with respect to both of the frequencies $k \pi / M$ and $(k+1) \pi / M$ to suppress both ICI components. Naturally, the ideal full-band channel equalization (resulting in constant amplitude and zero phase) implies both conditions. In our FBMC system, the equalization is performed at low rate, after filtering and decimation by $M$, and the mentioned two frequencies correspond to 0 and $\pi$, that is, the filtered and downsampled portion of $H_{\mathrm{ch}}\left(e^{j \omega}\right)$ in subchannel $k$ multiplied by the equalizer $C_{k}\left(e^{j \omega}\right)$ must fulfill the symmetry condition for zero ICI. In this case, the two symmetry conditions are equivalent (i.e., symmetric amplitude around 0 implies symmetric amplitude around $\pi$, and antisymmetric phase around 0 implies antisymmetric phase around $\pi$ ). The target is to approximate ideal channel equalization over the subchannel passband and transition bands with sufficient accuracy.

\subsubsection{ISI analysis}

In case of an ideal channel, the desired subchannel impulse response of the baseband model can be written as

$$
\tilde{t}_{k}[n]=\tilde{t}_{k}^{I}[n]+j \tilde{t}_{k}^{Q}[n]=t_{k}[n] e^{-j n k \pi / M} .
$$

For odd subchannels, a lowpass-to-highpass transformation has to be included in the model to get the actual response for the decimated filter bank, but the model above is suitable for analyzing all subchannels. Now the real part of the subchannel response with actual channel and equalizer can be written (see the appendix) as

$$
\begin{aligned}
\tilde{g}_{k}[n] & =\operatorname{Re}\left[\tilde{t}_{k}[n] * \tilde{r}_{k}[n]\right]=\operatorname{Re}\left[t_{0}[n] * \tilde{r}_{k}[n]\right] \\
& =t_{0}^{I}[n] * \operatorname{Re}\left[\tilde{r}_{k}[n]\right]-t_{0}^{Q}[n] * \operatorname{Im}\left[\tilde{r}_{k}[n]\right] .
\end{aligned}
$$

The conditions for suppressing ICI are also sufficient for suppressing the latter term of this equation. Furthermore, in case of PR filter bank design, $t_{0}^{I}[n]$ is a Nyquist pulse. Designing the channel equalizer to provide unit amplitude and zerophase response, a condition equivalent of having

$$
\operatorname{Re}\left[\tilde{r}_{k}[n]\right]=\delta[n]= \begin{cases}1, & n=0 \\ 0, & \text { otherwise }\end{cases}
$$

would suppress the ISI within the subchannel.
The above conditions were derived in the high-rate, fullband case, and if the conditions are fully satisfied, ISI within the subchannel and ICI from the lower and upper adjacent subchannels are completely eliminated. In practice, the equalization takes place at the decimated low sampling rate, and can be done only within the passband and transition band regions (assuming roll-off $\rho=1.0$ ). However, the ICI and ISI components outside the equalization band are proportional to the stopband attenuation of the subchannel filters and can be ignored.

\subsection{Optimization criteria for the equalizer coefficients}

Our interest is in low-complexity subcarrier equalizers, which do not necessarily provide responses very close to the ideal in all cases. Therefore, it is important to analyze the ICI and ISI effects with practical equalizers. This can be carried out most conveniently in frequency domain. In the baseband model, the lower and upper ICI spectrum magnitudes are

$$
\begin{aligned}
& \left|\widetilde{V}_{k}^{Q}\left(e^{j \omega}\right) \widetilde{R}_{k}^{Q}\left(e^{j \omega}\right)\right| \\
& =\left|V_{0}^{Q}\left(e^{j \omega}\right) \tilde{R}_{k}^{Q}\left(e^{j \omega}\right)\right| \\
& =\frac{M}{2}\left|H_{p}\left(e^{j(\omega-(\pi / 2 M))}\right) H_{p}\left(e^{j(\omega+(\pi / 2 M))}\right)\right| \cdot\left|\widetilde{R}_{k}^{Q}\left(e^{j \omega}\right)\right|, \\
& \left|\tilde{U}_{k}^{Q}\left(e^{j \omega}\right) \widetilde{R}_{k}^{Q}\left(e^{j(\omega+(\pi / M))}\right)\right| \\
& =\left|U_{2 M-1}^{Q}\left(e^{j \omega}\right) \widetilde{R}_{k}^{Q}\left(e^{j(\omega+(\pi / M))}\right)\right| \\
& =\frac{M}{2}\left|H_{p}\left(e^{j(\omega-(\pi / 2 M))}\right) H_{p}\left(e^{j(\omega t(\pi / 2 M))}\right)\right| \cdot\left|\widetilde{R}_{k}^{Q}\left(e^{j(\omega+(\pi / M))}\right)\right|,
\end{aligned}
$$

respectively. Here the upper-case symbols stand for the Fourier transforms of the impulse responses denoted by the corresponding lower-case symbols. The terms involving the two frequency shifted prototype frequency responses are the overall magnitude response for the crosstalk. $H_{p}\left(e^{j(\omega-(\pi / 2 M))}\right)$ appears here as the receive filter for the desired subchannel and $H_{p}\left(e^{j(\omega+(\pi / 2 M))}\right)$ denotes the response of the transmit filter of the contiguous (potentially interfering) subchannel. The actual frequency response includes phase terms, but based on the discussion in the previous subsection we know that, in the baseband model of the ideal channel case, all the cross-talk energy is in the imaginary part of the impulse response. The residual imaginary part of the equalized channel impulse response $\widetilde{r}_{k}^{Q}[n]$ determines how much of this cross-talk energy appears as ICI in detection. It can be calculated as a function of frequency for a given set of equalizer coefficients, assuming the required knowledge on the channel response is available. Now the ICI power for subchannel $k$ can be obtained with good accuracy by integrating over the transition bands in the baseband 
model

$$
\begin{aligned}
P_{k}^{I C I}= & \int_{-\pi / 2 M}^{\pi / 2 M} \frac{M^{2}}{4}\left|H_{p}\left(e^{j(\omega-(\pi / 2 M))}\right) H_{p}\left(e^{j(\omega+(\pi / 2 M))}\right)\right|^{2} \\
& \cdot\left|\widetilde{R}_{k}^{Q}\left(e^{j \omega}\right)\right|^{2} d \omega \\
+\int_{-\pi / 2 M}^{\pi / 2 M} & \frac{M^{2}}{4}\left|H_{p}\left(e^{j(\omega-(\pi / 2 M))}\right) H_{p}\left(e^{j(\omega+(\pi / 2 M))}\right)\right|^{2} \\
& \cdot\left|\widetilde{R}_{k}^{Q}\left(e^{j(\omega+(\pi / M))}\right)\right|^{2} d \omega .
\end{aligned}
$$

Also the ISI power can be calculated, as soon as the channel and equalizer responses are known, from the aliased spectrum of $\widetilde{G}_{k}\left(e^{j \omega}\right)$, as

$$
P_{k}^{I S I}=\int_{0}^{\pi / M}\left|M-\sum_{l=-1}^{1} \tilde{G}_{k}\left(e^{j(\omega+(l \pi / M))}\right)\right|^{2} d \omega .
$$

Here, the Nyquist criterion in frequency domain is used: in ISI-free conditions, the folded spectrum of the overall subchannel response $\widetilde{G}_{k}\left(e^{j \omega}\right)$ adds up to a constant level $M$, a condition equivalent to overall impulse response being unity impulse. By calculating the difference between this ideal reference level and the actual spectrum, the spectrum resulting from the residual ISI can be extracted. Integration over this residual spectrum gives the ISI power, according to (21).

Typically, the pulse shape applied to the symbol detector, the slicer, is constrained to satisfy the Nyquist criterion. In the presence of ISI, this often requires from the receive filter (in this context, the term "receive filter" is assumed to include both the analysis filter and the equalizer) a gain that compensates for the channel loss and causes the noise power to be amplified. This is called noise enhancement. The subchannel noise gain can be calculated as

$$
\beta_{k}^{n}=\frac{1}{2 \pi} \int_{-\pi}^{\pi}\left|C_{k}\left(e^{j \omega}\right) H_{p}\left(e^{j((\omega \mp \pi / 2) / M)}\right)\right|^{2} d \omega,
$$

where $C_{k}\left(e^{j \omega}\right)$ is the response of the subchannel equalizer. The - and + signs are valid for even and odd subchannel indexes, respectively.

\subsection{Semianalytical performance evaluation}

The performance of the studied FBMC, using per-subcarrier equalization to combat multipath distortion, can be evaluated semianalytically according to the discussion above. The term "semianalytically" refers, in this context, to the fact that no actual signal needs to be generated for transmission. Instead, a frequency domain analysis of the distorting channel and the equalizer can be applied to derive the ICI and ISI power spectra and the noise enhancement involved. Based on $P_{k}^{I C I}, P_{k}^{I S I}$, and $\beta_{k}^{n}$, the overall signal to interference plus noise ratio(s) (SINR) for given $E_{b} / N_{0}$-value(s) can be obtained. Then, well-known formulas based on the Q-function [30] and Gray-coding assumption can be exploited to estimate the uncoded bit error-rate (BER) performance. This can further be averaged over a number of channel instances corresponding to a given power delay profile.

\section{LOW-COMPLEXITY POINTWISE PER-SUBCARRIER EQUALIZATION}

The known channel equalization solutions for FBMC suffer from insufficient performance, as in the case of the 0th-order ASCET [22], and/or from relatively high implementation complexity, as in the FIR filter based approach described, for example, by Hirosaki in [4]. To overcome these problems, a specific structure that equalizes at certain frequency points is considered. The pointwise equalization principle proceeds from the consideration that the subchannel equalizers are designed to equalize the channel optimally at certain frequency points within the subband. To be more precise, the coefficients of the equalizer are set such that, at all the considered frequency points, the equalizer amplitude response optimally approaches the inverse of the determined channel amplitude response and the equalizer phase response optimally approaches the negative of the determined channel phase response. Optimal equalization at all frequencies would implicitly fulfill the zero ICI conditions of (15), and the zero ISI condition of (18). In pointwise equalization, the optimal linear equalizer is approximated between the considered points and the residual ICI and ISI interference powers depend on the degree of inaccuracy with respect to the zero ICI/ISI conditions and can be measured using (20) and (21), respectively. On the other hand, the level of inaccuracy depends on the relation of the channel coherence bandwidth [31] to the size of the filter bank and the order of the pointwise per-subcarrier equalizer. For mildly frequency selective subband responses, low-complexity structures are sufficient to keep the residual ICI and ISI at tolerable levels.

Alternative optimization criteria are possible for the equalizer coefficients from the amplitude equalization point of view, namely, zero-forcing (ZF) and mean-squared error (MSE) criteria [30, 31]. The most straightforward approach is $\mathrm{ZF}$, where the coefficients are set such that the achieved equalizer response compensates the channel response exactly at the predetermined frequency points. The ZF criterion aims to minimize the $P_{k}^{I C I}$ and $P_{k}^{I S I}$, but ignores the effect of noise. Ultimately, the goal is to minimize the probability of decision errors. The MSE criterion tries to achieve this goal by making a tradeoff between the noise enhancement and residual ISI at the slicer input. The MSE criterion thus alleviates the noise enhancement problem of ZF and could provide improved performance for those subchannels that coincide with the deep notches in the channel frequency response. For high SNR, the MSE solution of the amplitude equalizer converges to that obtained by the ZF criterion.

\subsection{Complex FIR equalizer}

A straightforward way to perform equalization at certain frequency points within a subband is to use complex FIR filter (CFIR-SCE), an example structure of which is shown in Figure 4, that has the desired frequency response at those given points. In order to equalize for example at three 


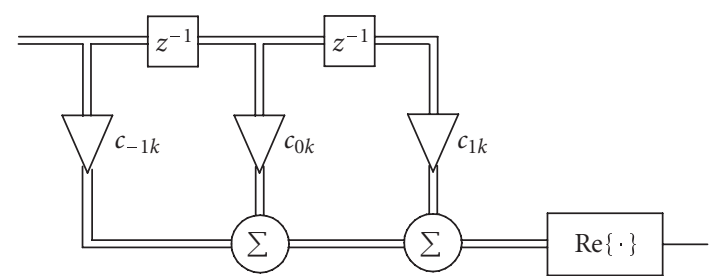

FIgURE 4: An example structure of the CFIR-SCE subcarrier equalizer.

frequency points, a 3-tap complex FIR with noncausal transfer function

$$
H_{\text {CFIR }-\operatorname{SCE}}(z)=c_{-1} z+c_{0}+c_{1} z^{-1}
$$

offers the needed degrees of freedom. The equalizer coefficients are calculated by evaluating the transfer function, which is set to the desired response, at the chosen frequency points and setting up an equation system that is solved for the coefficients.

\subsection{Amplitude-phase equalizer}

We consider a linear equalizer structure consisting of an allpass phase correction section and a linear-phase amplitude equalizer section. This structure is applied to each complex subchannel signal for separately adjusting the amplitude and phase. This particular structure makes it possible to independently design the amplitude equalization and phase equalization parts, leading to simple algorithms for optimizing the equalizer coefficients. The orders of the equalizer stages are chosen to obtain a low-complexity solution. A few variants of the filter structure have been studied and will be described in the following.

An example structure of the AP-SCE equalizer is illustrated in detail in Figure 5. In this case, each subchannel equalizer comprises a cascade of a first-order complex allpass filter, a phase rotator combined with the operation of taking the real part of the signal, and a first-order real allpass filter for compensating the phase distortion. The structure, moreover, consists of a symmetric 5-tap FIR filter for compensating the amplitude distortion. Note that the operation of taking the real part of the signal for detection is moved before the real allpass phase correction stage. This does not affect the output of the AP-SCE, but reduces its implementation complexity.

The transfer functions of the real and complex first-order allpass filters are given by

$$
\begin{aligned}
& H_{r}(z)=\frac{1+b_{r} z}{1+b_{r} z^{-1}}, \\
& H_{c}(z)=\frac{1-j b_{c} z}{1+j b_{c} z^{-1}},
\end{aligned}
$$

respectively. In practice, these filters are realized in the causal form as $z^{-1} H .(z)$, but the above noncausal forms simplify the following analysis. For the considered example structure, the overall phase response of the AP-SCE phase correction section (for the $k$ th subchannel) can be derived from (24) and (25)

$$
\begin{aligned}
\arg \left[H_{\mathrm{peq}}\left(e^{j \omega}\right)\right]= & \arg \left[e^{j \varphi_{0 k}} \cdot H_{c}\left(e^{j \omega}\right) \cdot H_{r}\left(e^{j \omega}\right)\right] \\
= & \varphi_{0 k}+2 \arctan \left(\frac{-b_{c k} \cos \omega}{1+b_{c k} \sin \omega}\right) \\
& +2 \arctan \left(\frac{b_{r k} \sin \omega}{1+b_{r k} \cos \omega}\right) .
\end{aligned}
$$

In a similar manner, we can express the transfer function of the amplitude equalizer section in a noncausal form as

$$
H_{\mathrm{aeq}}(z)=a_{2} z^{2}+a_{1} z+a_{0}+a_{1} z^{-1}+a_{2} z^{-2},
$$

from which the equalizer magnitude response for the $k$ th subchannel is obtained

$$
\left|H_{\mathrm{aeq}}\left(e^{j \omega}\right)\right|=\left|a_{0 k}+2 a_{1 k} \cos \omega+2 a_{2 k} \cos 2 \omega\right| .
$$

\subsection{Low-complexity AP-SCE and CFIR-SCE}

Case 1. The subchannel equalization is based on a single frequency point located at the center frequency of a specific subchannel, at $\pm \pi / 2$ at the low sampling rate. Here the + sign is valid for the even and the - sign is valid for the odd subchannel indexes, respectively. In this case, the associated phase equalizer only has to comprise a complex coefficient $e^{j \varphi_{0 k}}$ for phase rotation. The amplitude equalizer is reduced to just one real coefficient as a scaling factor. This case corresponds to the 0th-order ASCET or a single-tap CFIR-SCE.

Case 2. Here, equalization at two frequency points located at the edges of the passband of a specific subchannel, at $\omega=0$ and $\omega= \pm \pi$, is expected to be sufficient. The + and - signs are again valid for the even and odd subchannels, respectively. In this case, the associated equalizer has to comprise, in addition to the complex coefficient $e^{j \varphi_{0 k}}$, the first-order complex allpass filter as the phase equalizer, and a symmetric 3tap FIR filter as the amplitude equalizer. Compared to the equalizer structure of Figure 5, the real allpass filter is omitted and the length of the 5-tap FIR filter is reduced to 3. In the CFIR-SCE approach, two taps are used.

Case 3. Here, three frequency points are used for channel equalization. One frequency point is located at the center of the subchannel frequency band, at $\omega= \pm \pi / 2$, and two frequency points are located at the passband edges of the subchannel, at $\omega=0$ and $\omega= \pm \pi$. In this case, the associated equalizer has to comprise all the components of the equalizer structure depicted in Figure 5. In the CFIR-SCE structure of Figure 4, all three taps are used.

Mixed cases of phase and amplitude equalization. Naturally, also mixed cases of AP-SCE are possible, in which a different number of frequency points within a subband are considered for the compensation of phase and amplitude distortion. For 


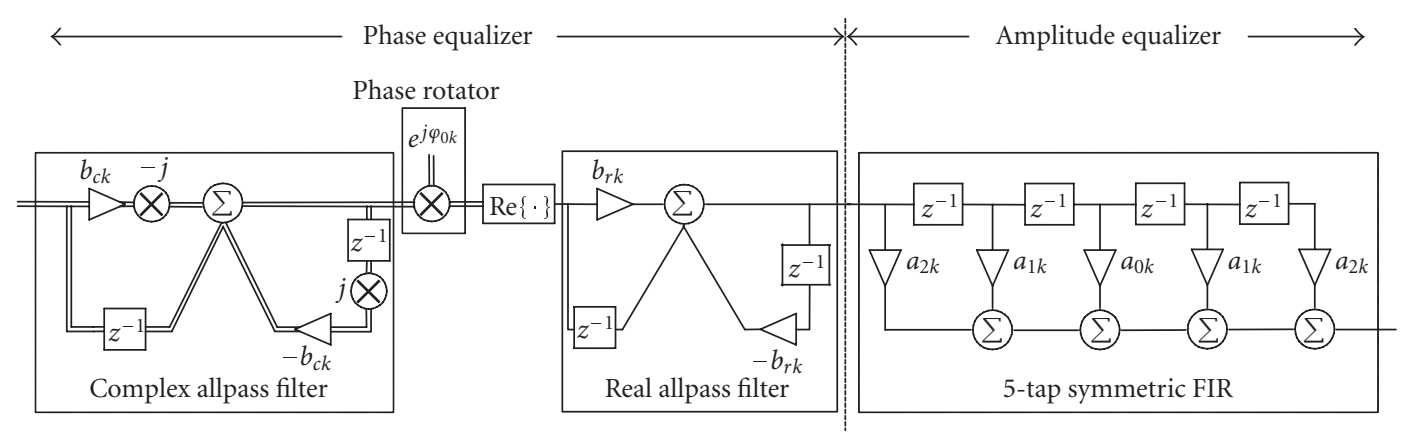

FIgURE 5: An example structure of the AP-SCE subcarrier equalizer.

example, Case 3 phase equalization could be combined with Case 2 amplitude correction and so forth. Ideally, the number of frequency points considered within each subchannel is not fixed in advance, but can be individually determined for each subchannel based on the frequency domain channel estimates of each data block. This enables the structure of each subchannel equalizer to be controlled such that the associated subchannel response is equalized optimally at the minimum number of frequency points which can be expected to result in sufficient performance. The CFIR-SCE cannot provide such mixed cases.

Also further cases could be considered since additional frequency points are expected to result in better performance when the subband channel response is more selective. However, this comes at the cost of increased complexity in processing the data samples and much more complicated formulas for obtaining the equalizer coefficients.

For Case 3 structure, CFIR-SCE and AP-SCE equalizer coefficients can be calculated by evaluating (23) and (26), and (28), respectively, at the frequency points of interest, setting them equal to the target values, and solving the resulting system of equations for the equalizer coefficients:

CFIR-SCE:

$$
\begin{aligned}
c_{-1 k} & =\frac{\gamma}{4}\left(\left(\chi_{0 k}-\chi_{2 k}\right) \mp j\left(2 \chi_{1 k}-\chi_{0 k}-\chi_{2 k}\right)\right), \\
c_{0 k} & =\frac{\gamma}{2}\left(\chi_{0 k}+\chi_{2 k}\right), \\
c_{1 k} & =\frac{\gamma}{4}\left(\left(\chi_{0 k}-\chi_{2 k}\right) \pm j\left(2 \chi_{1 k}-\chi_{0 k}-\chi_{2 k}\right)\right) ;
\end{aligned}
$$

AP-SCE:

$$
\begin{aligned}
\varphi_{0 k} & =\frac{\xi_{0 k}+\xi_{2 k}}{2}, & a_{0 k} & =\frac{\gamma}{4}\left(\epsilon_{0 k}+2 \epsilon_{1 k}+\epsilon_{2 k}\right), \\
b_{c k} & = \pm \tan \left(\frac{\xi_{2 k}-\xi_{0 k}}{4}\right), & a_{1 k} & = \pm \frac{\gamma}{4}\left(\epsilon_{0 k}-\epsilon_{2 k}\right), \\
b_{r k} & = \pm \tan \left(\frac{\xi_{1 k}-\varphi_{0 k}}{2}\right), & a_{2 k} & =\frac{\gamma}{8}\left(\epsilon_{0 k}-2 \epsilon_{1 k}+\epsilon_{2 k}\right) .
\end{aligned}
$$

Here the \pm signs are again for the even/odd subchannels, respectively, and $\chi_{i k}, \xi_{i k}$, and $\epsilon_{i k}, i=0, \ldots, 2$, are the complex target response, the target phase, and amplitude response values at the three considered frequency points for subchannel $k$. The value $i=1$ corresponds to the subchannel center frequency whereas values $i=0$ and $i=2$ refer to the lower and upper passband edge frequencies, respectively. With MSE criterion,

$$
\begin{aligned}
& \chi_{i k}=\frac{H_{\mathrm{ch}}\left(e^{j(2 k+i)(\pi / 2 M)}\right)^{*}}{\left|H_{\mathrm{ch}}\left(e^{j(2 k+i)(\pi / 2 M)}\right)\right|^{2}+\eta}, \\
& \xi_{i k}=\arg \left(\chi_{i k}\right), \quad \epsilon_{i k}=\left|\chi_{i k}\right|,
\end{aligned}
$$

where $H_{\mathrm{ch}}$ is the channel frequency response in the baseband model of the overall system. The effect of noise enhancement is incorporated into the solution of the equalizer parameters using the noise-to-signal ratio $\eta$ and a scaling factor $\gamma=3 / \sum_{i=0}^{2} \chi_{i k} H_{\mathrm{ch}}\left(e^{j(2 k+i)(\pi / 2 M)}\right)$ that normalizes the subchannel signal power to avoid any scaling in the symbol values used for detection. In the case of ZF criterion, $\eta=0$ and $\gamma=1$.

The operation of the ZF-optimized amplitude and phase equalizer sections of Case 3 AP-SCE are illustrated with randomly selected subchannel responses in Figures 6 and 7, respectively.

In Case 2, MSE-optimized coefficients for CFIR-SCE and AP-SCE amplitude equalizer can be calculated as

$$
\begin{aligned}
c_{0 k} & =\frac{\gamma}{2}\left(\chi_{0 k}+\chi_{2 k}\right), & a_{0 k} & =\frac{\gamma}{2}\left(\epsilon_{0 k}+\epsilon_{2 k}\right), \\
c_{1 k} & = \pm \frac{\gamma}{2}\left(\chi_{0 k}-\chi_{2 k}\right), & a_{1 k} & = \pm \frac{\gamma}{4}\left(\epsilon_{0 k}-\epsilon_{2 k}\right),
\end{aligned}
$$

where $\gamma=2 /\left(\chi_{0 k} H_{\mathrm{ch}}\left(e^{j(k \pi / M)}\right)+\chi_{2 k} H_{\mathrm{ch}}\left(e^{j(2 k+2)(\pi / 2 M)}\right)\right)$. The AP-SCE phase equalizer coefficients $\varphi_{0 k}$ and $b_{c k}$ can be obtained as in Case 3.

Case 1 equalizers are obtained as special cases of the used structures, including only a single complex coefficient for CFIR-SCE and an amplitude scaling factor and a phase rotator for AP-SCE. It is natural to calculate these coefficients based on the frequency response values at the subchannel center frequencies, that is,

$$
\begin{gathered}
c_{0 k}=\chi_{1 k}, \\
a_{0 k}=\left|\chi_{1 k}\right|, \quad \varphi_{0 k}=\arg \left(\chi_{1 k}\right),
\end{gathered}
$$

with $\eta=0$, since MSE and ZF solutions are the same. 


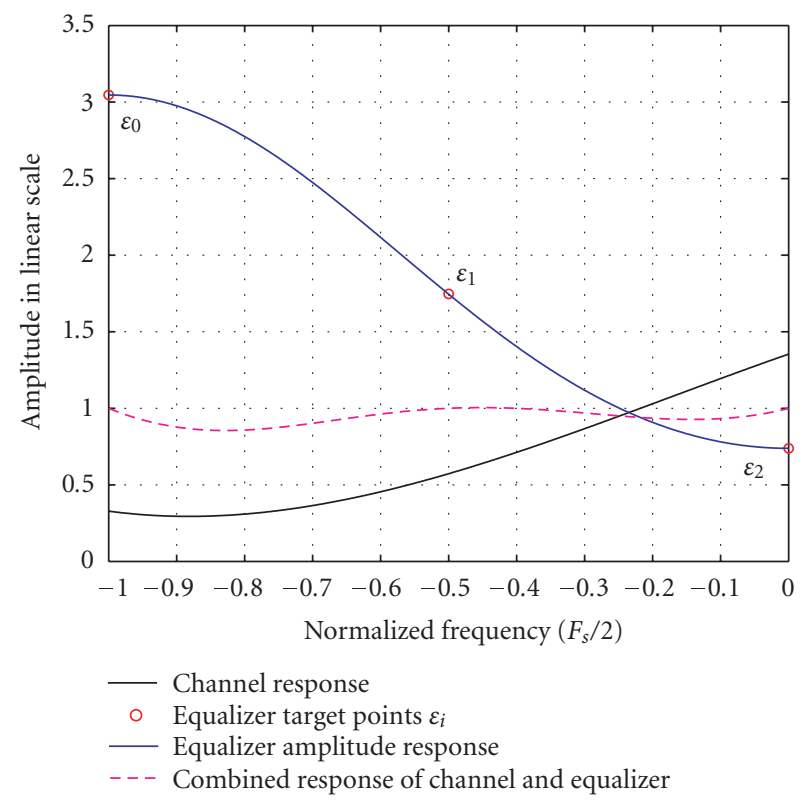

Figure 6: Operation of the ZF-optimized Case 3 amplitude equalizer section.

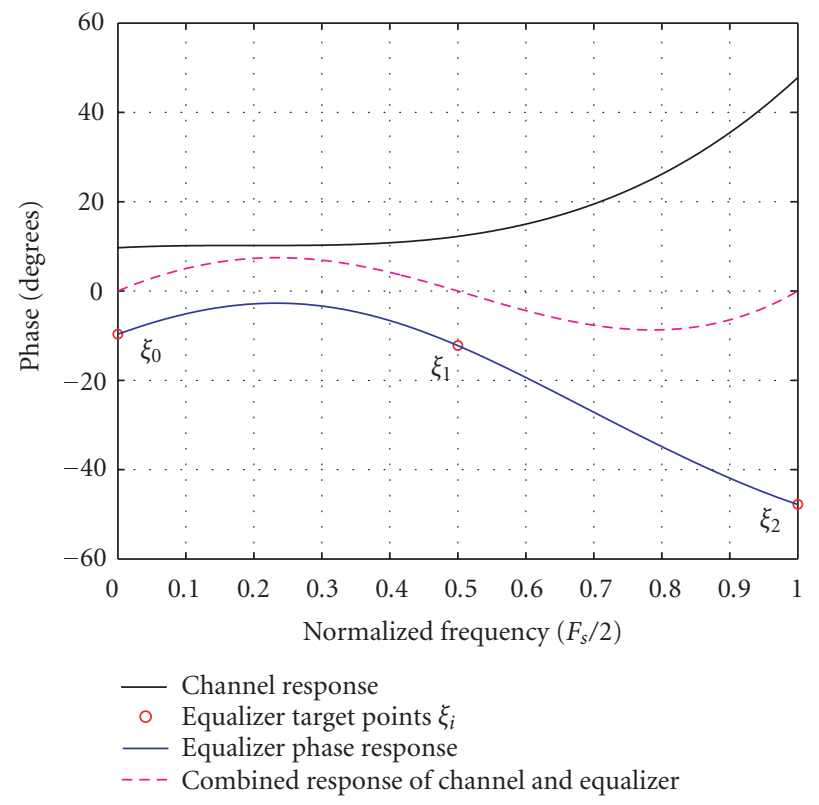

Figure 7: Operation of the Case 3 phase equalizer section.

\section{NUMERICAL RESULTS}

The performance of the low-complexity subcarrier equalizers was evaluated with different number of subchannels both semianalytically and using full simulations in time domain. First, basic results are reported to illustrate how the performance depends on the number of subcarriers and the equalizer design case. Also the reliability of the semianalytical model is examined and the differences between ZF and MSE criteria are compared. Finally, more complete simulations with error control coding are reported and compared to an OFDM reference in a realistic simulation environment. Also sensitivity to timing and frequency offsets and performance with practical transmitter power amplifiers are investigated. We consider equally spaced real 2-PAM, 4-PAM, and 8-PAM constellations for FBMC and complex square-constellations QPSK, 16-QAM, and 64-QAM in the OFDM case.

\subsection{Semianalytical performance evaluation}

Semianalytical simulations were carried out with the Vehicular-A power delay profile (PDP), defined by the recommendations of the ITU [32], for a $20 \mathrm{MHz}$ signal bandwidth. These simulations were performed in quasi-static conditions, that is, the channel was time-invariant during each transmitted frame. Perfect channel information was assumed. In all the simulations, the average channel power gain was scaled to unity. Performance was tested with filter banks consisting of $2 M=\{64,128,256\}$ subchannels. The filter bank designs used roll-off $\rho=1.0$ and overlapping factor $K=5$ resulting in about $50 \mathrm{~dB}$ stopband attenuation. The statistics are based on 2000 frame transmissions for each of which an independent channel realization was considered. The semianalytical results were obtained by calculating the subcarrierwise ICI and ISI powers $P_{k}^{I C I}$ and $P_{k}^{I S I}$, respectively, together with noise gains $\beta_{k}^{n}$ for $k=0,1, \ldots, 2 M-1$. These were then used to determine the subcarrierwise SINR-values, as a function of channel $E_{b} / N_{0}$-values, for all the channel instances. The uncoded BER results were obtained for 2-, 4-, and 8-PAM modulations by evaluating first the theoretical subcarrierwise BERs based on the SINR-values using the Qfunction and Gray-coding assumption, and finally averaging the BER over all the subchannels and 2000 channel instances.

\subsubsection{Basic results for AP-SCE}

The comparison in Figure 8(a) for ZF 4-PAM shows that the time domain simulation-based (Sim) and semi-analytic model-based (SA) results match quite well. This encourages to carry out system performance evaluations, especially in the algorithm development phase, mostly using the semianalytical approach, which is computationally much faster. Time domain simulation results in Figure 8(b) for 4-PAM indicate that the performance difference of ZF and MSE criteria is rather small. Figures $8(\mathrm{c})$ and $8(\mathrm{~d})$ show the semi-analytic results for 2-PAM and 8-PAM, respectively, using the ZF criterion. It can be observed that higher-order AP-SCE improves the equalizer performance significantly, allowing the use of a lower number of subcarriers. Also ideal OFDM performance (without guard interval overhead) is shown as a reference. With the aid of the AP-SCE equalizer, the performance of FBMC with a modest number of subcarriers can be made to approach that of the ideal OFDM.

\subsubsection{Comparison of CFIR-FBMC and AP-FBMC}

In the other simulations, it is assumed that the receiver is time-synchronized such that the first path corresponds to 


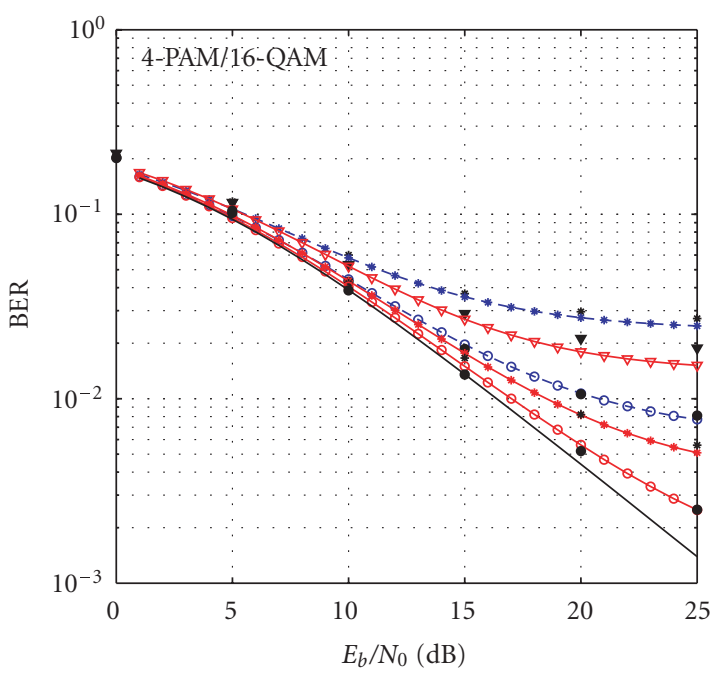

* $\quad 2 M=128$, case 1, Sim $* 2 M=128$, case 3, SA

-*- $2 M=128$, case $1, \mathrm{SA} \quad * 2 M=128$, case 3 , Sim

$\nabla 2 M=64$, case 3 , Sim $\bigcirc 2 M=256$, case 3 , SA

$\nabla 2 M=64$, case 3, SA $\quad-2 M=256$, case 3, Sim

$-\ominus-2 M=256$, case 1, SA

- $2 M=256$, case 1, Sim

(a)

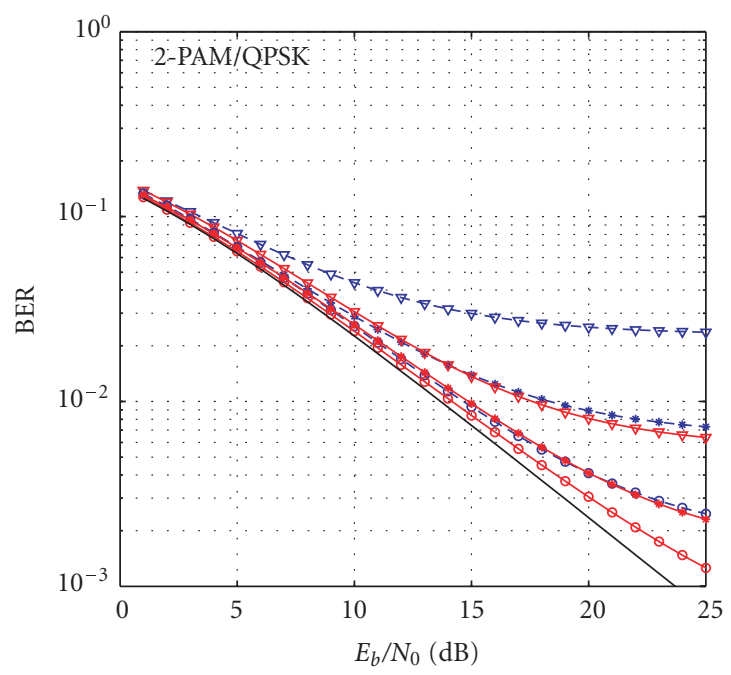

$$
\begin{aligned}
& -\nabla-2 M=64 \text {, case } 1 \rightarrow-2 M=128 \text {, case } 3 \\
& \text { - * } 2 M=128 \text {, case } 1 \multimap 2 M=256 \text {, case } 3 \\
& \rightarrow 2 M=64 \text {, case } 3 \longrightarrow \text { Ideal OFDM }
\end{aligned}
$$$$
-\ominus-2 M=256 \text {, case } 1
$$

(c)

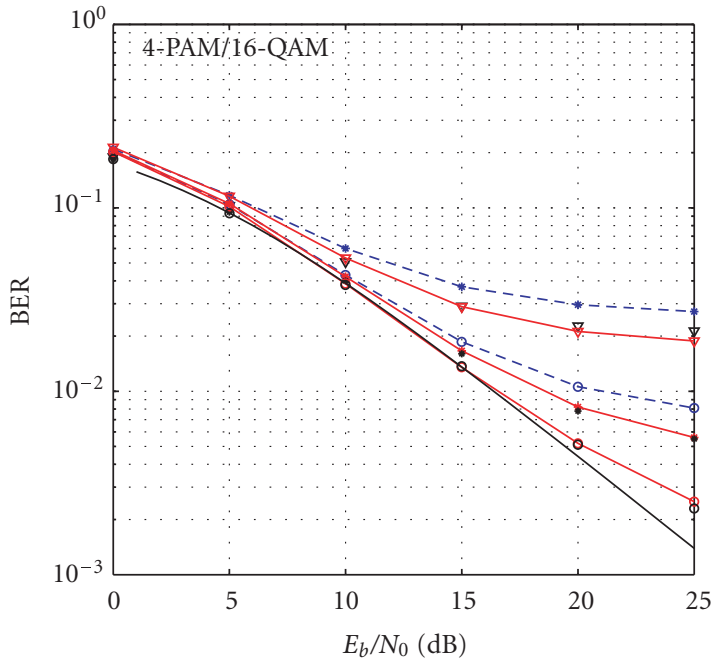

$$
\begin{aligned}
-*-2 M & =128, \text { case } 1, \mathrm{ZF} \quad * \quad 2 M=128, \text { case } 3, \text { MSE } \\
\nabla \quad 2 M & =64, \text { case } 3, \mathrm{MSE} \quad-\quad 2 M=256, \text { case } 3, \mathrm{ZF} \\
\nabla-2 M & =64, \text { case } 3, \mathrm{ZF} \quad \circ \quad 2 M=256, \text { case } 3, \mathrm{MSE} \\
-\ominus-2 M & =256, \text { case } 1, \mathrm{ZF} \quad-\quad \text { Ideal OFDM } \\
-2 M & =128, \text { case } 3, \mathrm{ZF}
\end{aligned}
$$

(b)

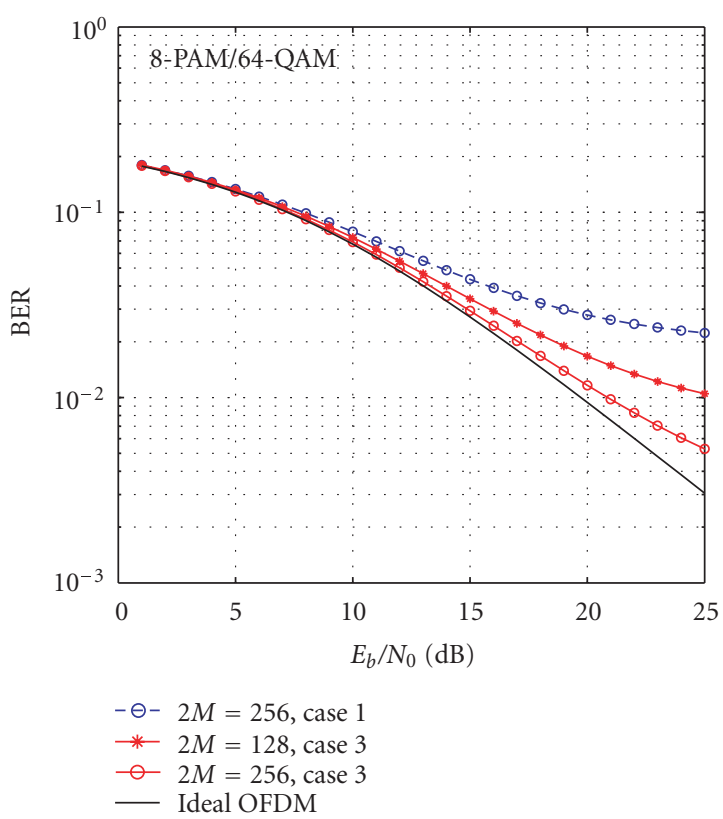

(d)

FIGURE 8: Uncoded BER results for AP-SCE with a quasi-static ITU-R Vehicular-A channel model and $20 \mathrm{MHz}$ bandwidth. (a) Comparison of time domain simulations (Sim) and semi-analytic model (SA) for ZF 4-PAM. (b) Comparison of ZF and MSE criteria with 4-PAM based on time domain simulations. (c) Semi-analytic performance of ZF 2-PAM. (d) Semi-analytic performance of ZF 8-PAM. Ideal OFDM (using corresponding square-constellation QAM, without guard interval overhead) included in all figures as a reference.

zero delay. Figure 9, however, shows a semi-analytic BER comparison of the two subcarrier equalizer structures for $2 M=256$ subchannels when the effect of time synchronization error is considered. Simulations were carried out with a quasi-static channel model based on the extended ITU-R
Vehicular-A PDP [33]. Simulation result statistics are based on 2000 independent channel instances of this model and the MSE criterion was used in the derivation of the amplitude equalizer coefficients. Figure 9 shows the performance in two cases: with delays of 0 and 64 samples, corresponding to 0 


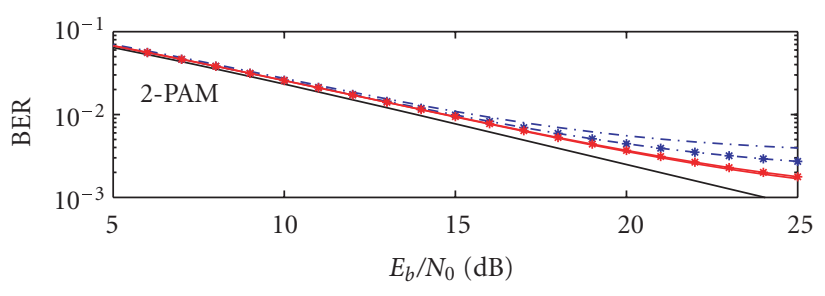

(a)

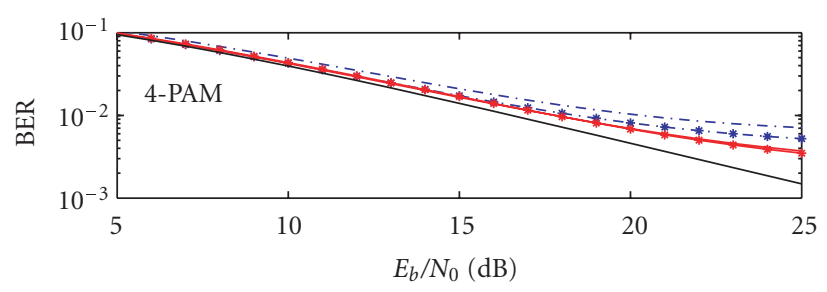

(b)
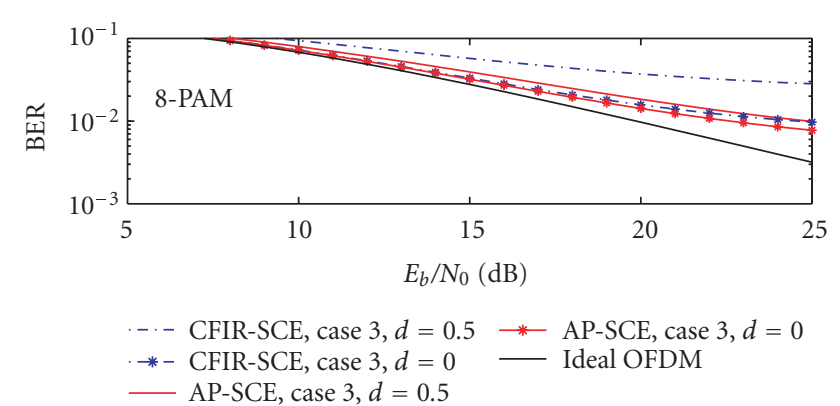

(c)

Figure 9: Semi-analytic BER in AP-SCE and CFIR-SCE. Parameter $d=$ timing offset/subcarrier symbol interval.

and $50 \%$ of the subcarrier symbol interval, respectively. It is seen that with 0 timing offset, CFIR-SCE and AP-SCE have very similar performance. However, AP-SCE is clearly more robust in the presence of timing offset. Especially with highorder modulations, the performance of CFIR-SCE is significantly degraded when the timing error approaches half of the subcarrier symbol interval. AP-SCE is very robust in this sense, and the results demonstrate that FBMC with AP-SCE can be operated without timing synchronization prior to the receiver filter bank.

Figure 10 shows the signal-to-interference ratio (SIR) performance in case of an ideal channel with timing offset only. Here, Case 2 AP-SCE includes only the first-order complex allpass and phase rotation; the real allpass does not have any effect in this case. Figure 10 was obtained in the $2 M=256$ subcarrier case, but it was observed that with other filter bank sizes, the behavior in terms of relative timing offset is very similar. It is seen that Case 3 CFIR-SCE gives clearly better performance than simple phase rotation (Case 1), and with timing offsets approaching half of the symbol interval, Case $2 \mathrm{AP}-\mathrm{SCE}$ has $3 \mathrm{~dB}$ better performance than Case 3 CFIR-SCE. This is in accordance with the findings in [34], where it is observed that allpass IIR structures

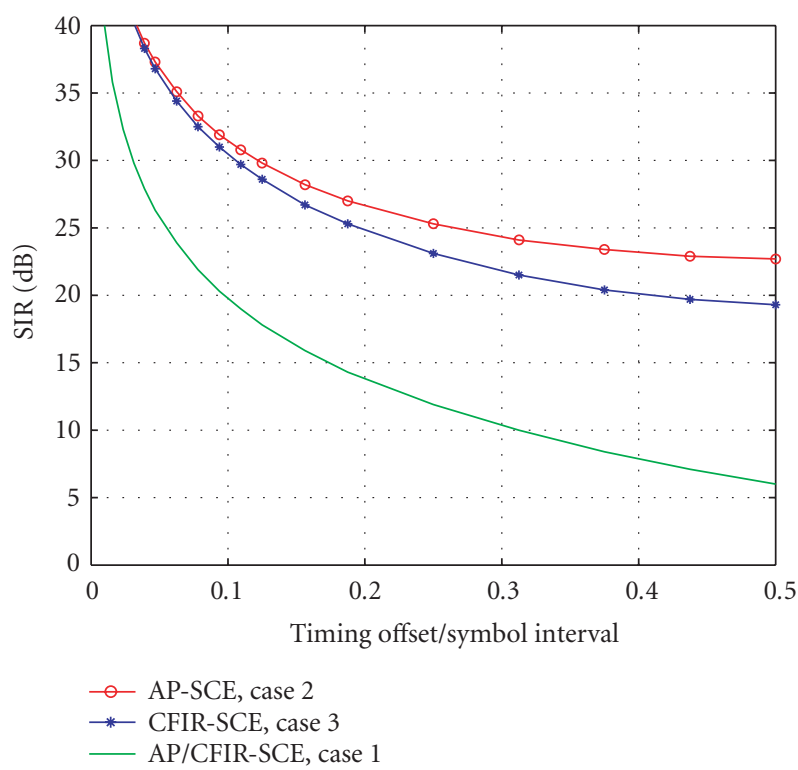

FIGURE 10: Semi-analytic SIR due to timing phase offset in AP-SCE and CFIR-SCE in an ideal channel.

provide performance gain in fractional delay compensation compared to FIR structures with similar complexity.

\subsection{Performance comparisons with channel coding}

\subsubsection{Channel model, system parameters, and OFDM reference}

We have also carried out full simulations in time domain comparing cyclic prefix OFDM and FBMC. It was of particular interest to evaluate the performance of FBMC with AP-SCE and CFIR-SCE per-subcarrier equalizers and to explore the potential spectral efficiency gain. Time-variant radio channel impairments were modeled based on the extended ITU-R Vehicular-A PDP [33] (maximum excess delay of $2.51 \mu \mathrm{s})$. This upgraded channel model has been shown to improve the frequency correlation properties when compared to the original PDP, making it better suited for evaluation of wideband transmission with frequencydependent characteristics. Mobile velocity of $50 \mathrm{~km} / \mathrm{h}$ and carrier frequency of $5 \mathrm{GHz}$ were assumed. With sampling rate of $26.88 \mathrm{MHz}(7 \times$ WCDMA chip rate), 616 subcarriers of 1024 in OFDM and 84/168/672 subchannels of 128/256/1024 in FBMC were activated to obtain systems with the same effective bandwidth of $18 \mathrm{MHz}$ (at $40 \mathrm{~dB}$ below passband level). This corresponds to subchannel bandwidths of $26.25 \mathrm{kHz}$ and $210 / 105 / 26.25 \mathrm{kHz}$, respectively. 2-, 4-, and 8-PAM modulations were considered for FBMC whereas QPSK, 16-QAM, and 64-QAM were used for OFDM. The FBMC design used roll-off $\rho=1.0$ and overlapping factor $K=5$ resulting in a stopband attenuation (defined as the level of the highest sidelobe) of about $50 \mathrm{~dB}$ (for 2PAM/QPSK comparison also $K=3$ was considered, giving stopband attenuation of about $38 \mathrm{~dB}$ ). Channel coding 
was performed using low-density parity check (LDPC) coding [35]. The maximum number of iterations in iterative decoding was set to ten. About $10 \%$ overhead for pilot carriers is assumed in OFDM and similar overhead for training sequences in FBMC. OFDM has $41.67 \mu$ s overall symbol duration, with $2.53 \mu$ s guard interval and $1.04 \mu$ s raised-cosine roll-off for spectral shaping. Both systems have a single zero power subcarrier in the middle of the spectrum to facilitate receiver implementation. The information bit rates in the two systems were approximately matched using code rates of $R=3 / 4$ and $R=2 / 3$ for OFDM and FBMC, respectively. Bits for a single frame to be transmitted were coded in blocks of 3348 and 3990 bits, respectively, after which all the coded bits of a frame were randomly interleaved before bits-to-symbols and symbols-to-subcarriers mappings. The resulting number of source bits in a fixed frame duration of $250 \mu$ s are 5022 and 5320 for QPSK/OFDM and 2-PAM/FBMC, respectively. Ideal channel estimation was assumed for both OFDM and FBMC modulations. Simulation result statistics are based on 5000 transmitted frames for each of which an independent realization of the channel model was applied. MSE optimization criterion was used to derive the amplitude equalizer parameters.

\subsubsection{Coded results}

Figures 11(a), 11(b), and 11(c) show the obtained results for 2-PAM/QPSK, 4-PAM/16-QAM, and 8-PAM/64-QAM comparisons, respectively. Coded frame error rate (FER) and BER are shown as a function of required energy per source bit to noise spectral density-ratio. Due to the absence of time domain guard interval and reduced frequency domain guardbands, higher spectral efficiency in FBMC is achieved. This excess transmission capacity can be used to transmit more redundant data (lower coding rate) while maintaining similar information data rate compared to OFDM. This turns into favor of FBMC in the FER/BER performance comparison as somewhat less energy in FBMC is sufficient to result in similar error probability compared to OFDM. Alignment of the performance curves for $K=3$ and $K=5$ in Figure 11(a) indicates that at least in narrowband interference-free conditions, FBMC design with $K=3$ (and possibly even $K=$ 2 ) provides sufficient performance with reduced complexity compared to $K=5$.

\subsubsection{Effect of AP-SCE structure and parameters}

The ability of AP-SCE/CFIR-SCE equalizer to compensate for mildly frequency selective subchannel responses is clearly visible in the simulation results. FBMC of $2 M=256$ subchannels with Case 3 AP-SCE/CFIR-SCE follows the performance curves obtained with the structure consisting of $2 M=1024$ subchannels with Case 1 equalizer. So, great reduction in the number of subchannels required to cover the $18 \mathrm{MHz}$ signal band can be achieved with higher-order AP-SCE/CFIR-SCE structures. In case of 2-PAM modulation even a filter bank with $2 M=128$ subchannels can be considered. For 4-PAM and 8-PAM, 2M = 256 subchannels are required to keep the performance benefit with respect to the OFDM reference.

\subsection{Performance with nonlinear power amplifier}

The ratio between the maximum instantaneous power of a signal and its mean power (PAPR) is proportional to the number of subcarriers and also depends on the modulation constellation used. This is a matter of concern when the signal passes through a nonlinear device such as the power amplifier (PA). In this situation, signal components of different instantaneous power might be amplified differently, introducing distortion to the signal and causing spectral regrowth to the bands adjacent to the signal. In this section, we focus on the spectral regrowth caused by a PA on FBMC and OFDM with similar parameters as in the time domain BER simulations. We apply time domain raised-cosine windowing of 28 samples to the OFDM signal in order to assure attenuation of $40 \mathrm{~dB}$ for the signal at $9 \mathrm{MHz}$ from the carrier frequency. Therefore, the overall $40 \mathrm{~dB}$ bandwidth for OFDM and FBMC is $18 \mathrm{MHz}$. The PA follows the solid state power amplifier (SSPA) model that can be found in [36]. Only amplitude nonlinearity is taken into account. The amplitude gain is given by

$$
p_{o}=\frac{p_{i}}{\sqrt{1+\left(p_{i} / p_{\mathrm{sat}}\right)^{2}}}
$$

where $p_{i}$ and $p_{o}$ are the amplitude of the PA input signal and output signal, respectively, and $p_{\text {sat }}$ denotes the saturation voltage of the PA. The spectral regrowth is measured as a function of the input back-off (IBO) of the input signal at the amplifier. In Figure 12 we show the regrowth of the spectra of FBMC (dashed lines) and OFDM (continuous lines). For FBMC we simulate IBOs that are $1.2 \mathrm{~dB}$ higher than for OFDM. This reflects the fact that for a similar coded BER performance we can use an FBMC signal with $1.2 \mathrm{~dB}$ less power than OFDM. We can see from the figure, that it is of advantage to be able to use a weaker signal, since close to the desired passband we obtain less spectral regrowth. At more distant frequencies, the OFDM spectrum decays faster because the useful bandwidth is smaller than the useful bandwidth in FBMC (16.2 MHz versus $17.6 \mathrm{MHz}$ ). OFDM with a comparable useful bandwidth (672 active subcarriers) has a spectral decay profile similar to FBMC's. Moreover, at the same IBOs and same useful bandwidths, both systems show very similar regrowth curves.

\subsection{Frequency offset}

In multicarrier transmissions, frequency offsets (e.g., due to Doppler and inaccuracy of local oscillators in the transmission chain) introduce ICI. In case of a fixed frequency offset in OFDM, the SIR due to the resulting ICI is given by [37]

$$
\operatorname{SIR}=\frac{1}{(\sin (\pi \Delta f))^{2} \sum_{p=0, p \neq N_{c} / 2}^{N_{c}-1} 1 /\left\{N_{c} \sin \left(\pi(p+\Delta f) / N_{c}\right)\right\}^{2}},
$$




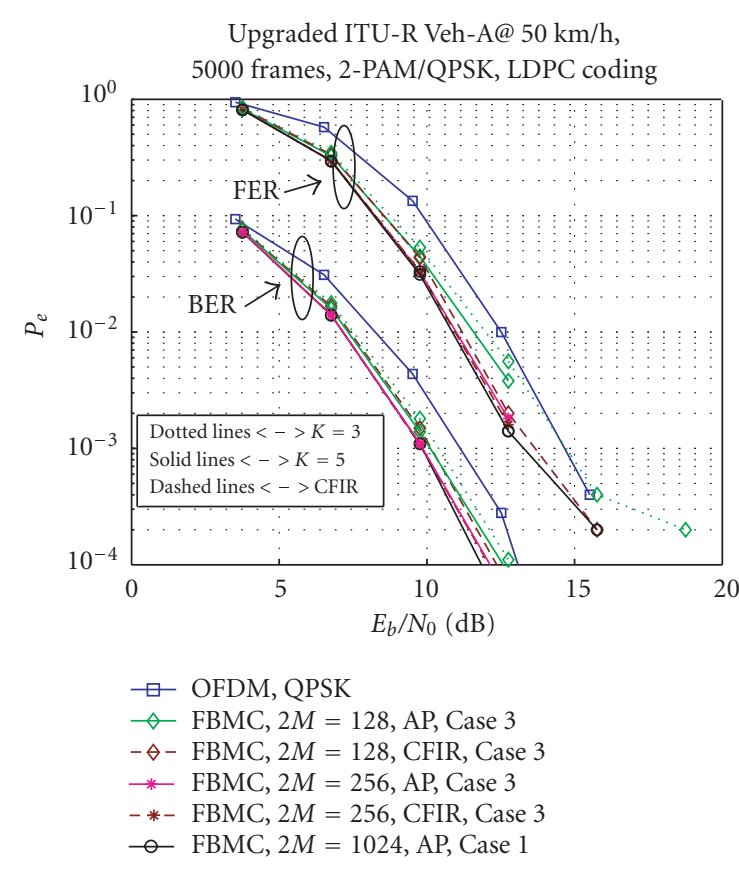

(a)

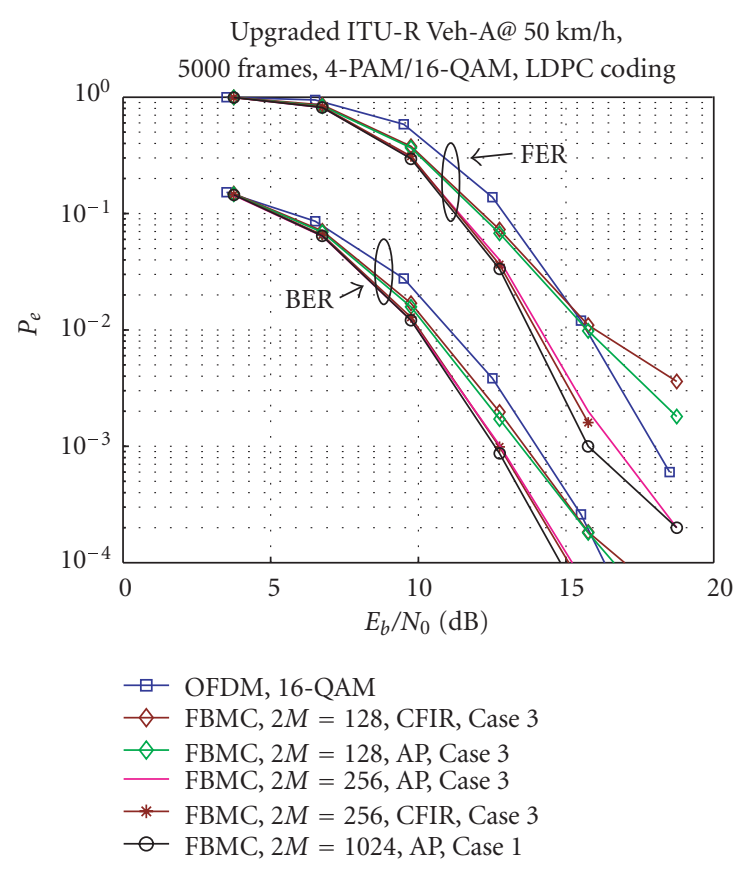

(b)

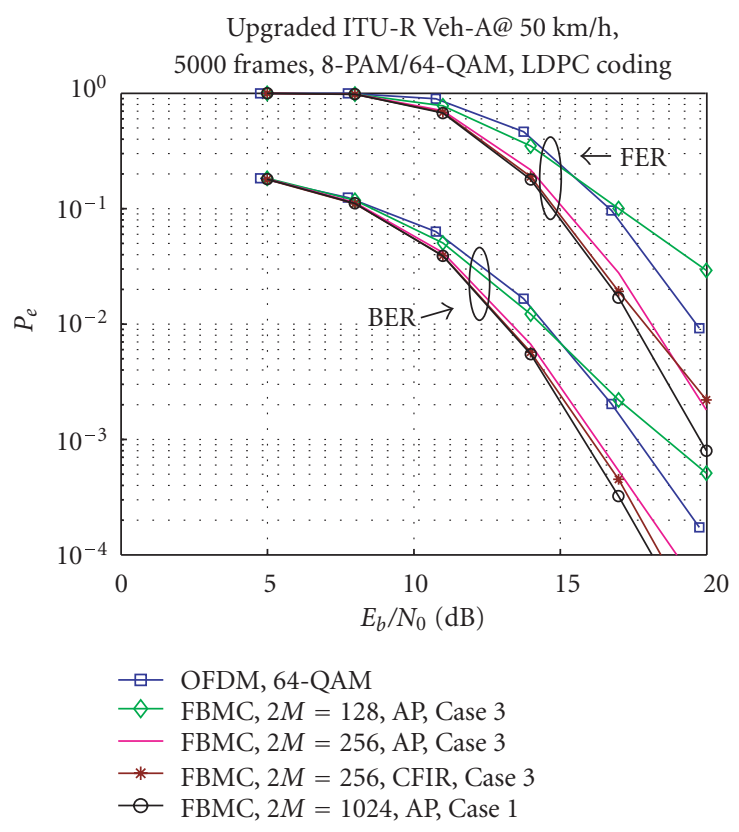

(c)

FIGURE 11: Coded FER and BER performance: (a) 2-PAM/FBMC and QPSK/OFDM; (b) 4-PAM/FBMC and 16-QAM/OFDM; and (c) 8PAM/FBMC and 64-QAM/OFDM.

where $N_{c}$ and $\Delta f$ are the number of subcarriers and frequency offset, respectively. The effects of frequency offsets in FBMC were tested with a simple simulation experiment by measuring the mean squared error in symbol detection with a set of fixed frequency offsets. The results are shown and compared to the OFDM performance in Figure 13. Here $N_{c}=256$ for both systems.
Basically, the frequency offset introduces a time-varying phase offset, which is common to all subcarriers. In the simulation, as well as in the analytical results for OFDM, the constant part of the common phase offset is assumed to be cancelled by the channel equalizer such that in the middle of each symbol the phase error of each subcarrier is zero. 


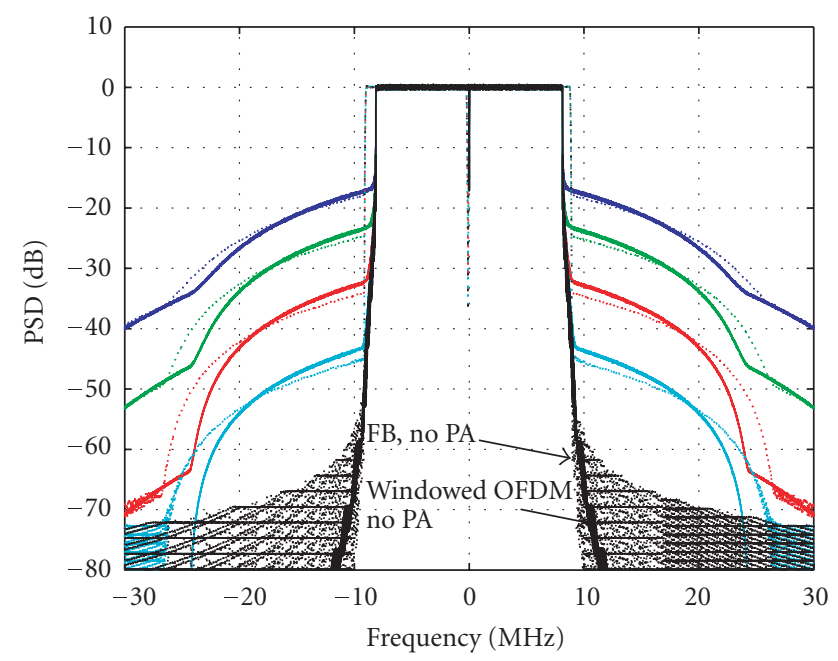

$\mathrm{FB}, \mathrm{IBO}=1.2 \mathrm{~dB} \quad$ - Windowed OFDM, IBO $=0 \mathrm{~dB}$
$\mathrm{FB}, \mathrm{IBO}=7.2 \mathrm{~dB} \quad$ Windowed OFDM, IBO $=6 \mathrm{~dB}$
$\mathrm{FB}, \mathrm{IBO}=13.2 \mathrm{~dB}$ - Windowed OFDM, IBO $=12 \mathrm{~dB}$
$\mathrm{FB}, \mathrm{IBO}=19.2 \mathrm{~dB}$ - Windowed OFDM, IBO $=18 \mathrm{~dB}$

FIGURE 12: Spectral regrowth due to PA nonlinearity.

It can be seen from Figure 13 that for a given relative (with respect to subcarrier spacing) frequency offset, the FBMC SIR performance is slightly better but within $2 \mathrm{~dB}$ from the OFDM performance. Since FBMC allows significantly wider subcarrier spacing, the relative frequency offsets are smaller, and there is a clear performance benefit for FBMC in terms of frequency offset effects. This indicates also a potential for better performance in case of fast fading.

\subsection{Complexity}

In this subsection, a rough evaluation of the computational complexity of FBMC is presented, using a simple complexity measure: the number of real multiplications required to detect a symbol. We focus on the receiver side where the OFDM FFT or FBMC analysis bank and the equalizer are the main processing blocks. Channel estimation and calculation of the equalizer coefficients are not included in this evaluation.

One of the most efficient algorithms for implementing DFT is the split radix FFT algorithm [18], taking $M\left(\log _{2}(M)-3\right)+4$ real multiplications for a block of $M$ complex samples. In the OFDM case, 3 real multipliers are enough to do the complex multiplication to equalize each of the used subcarriers.

In the FBMC case, the FFT-based algorithm presented in [26] is the most efficient one to implement the oversampled analysis bank in terms of multiplication rate. It requires $2 M\left(2 K-2+\log _{2}(M)\right)$ real multiplications for a block of $M$ high-rate samples. In an efficient implementation, the APSCE subcarrier equalizers take 2, 5, and 7 real multiplications in Cases 1, 2, and 3, respectively, per detected real symbol. Alternatively, the 3-tap CFIR-SCE structure takes 6 real multiplications per detected real symbol.

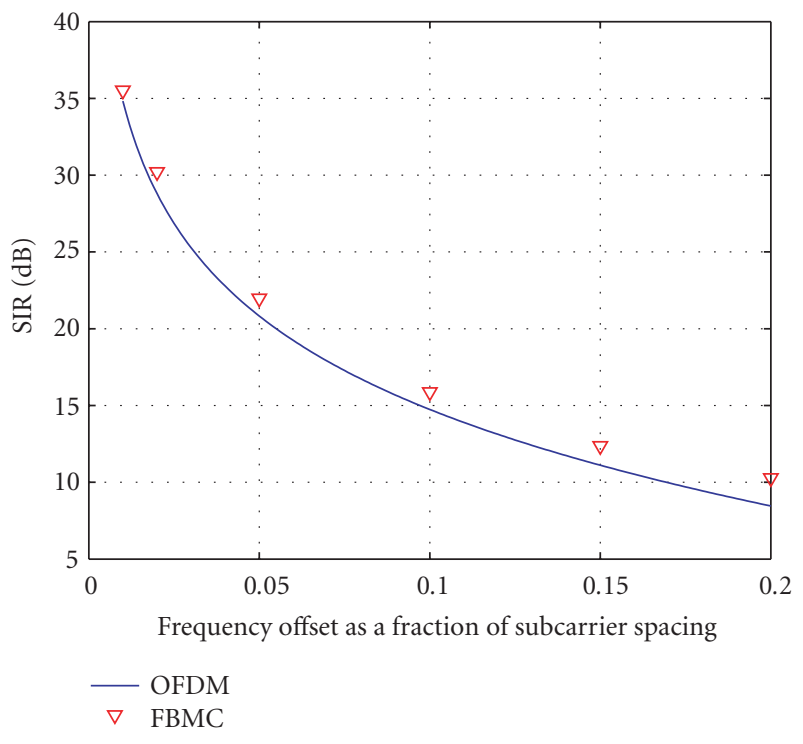

FIGURE 13: SIR due to frequency offset in OFDM and FBMC.

TABLE 1: Multiplications in receiver per one detected complex symbol in OFDM and per two detected real symbols in FBMC.

\begin{tabular}{lcc}
\hline & Case 1 & Case 3 \\
\hline OFDM, 1k-FFT & 10 & - \\
FBMC, $K=2,2 M=128$, AP-SCE & 20 & 30 \\
FBMC, $K=5,2 M=256$, AP-SCE & 34 & 44 \\
FBMC, $K=2,2 M=128$, CFIR-SCE & 20 & 28 \\
FBMC, $K=5,2 M=256$, CFIR-SCE & 34 & 42 \\
\hline
\end{tabular}

For a fair comparison, we calculate the overall number of multiplications per detected complex symbol in the OFDM case and per two detected real symbols in the FBMC case. For simplicity, it is assumed that all the subcarriers are in use. The resulting overall multiplication rates with the two extreme cases of FBMC-complexity are shown in Table 1.

It is observed that with this complexity measure, FBMC is more complex than the basic OFDM system. However, the implementation of FBMC is yet quite realistic with today's efficient digital signal processors or dedicated very large scale integration (VLSI) hardware. It is expected that there are a lot of possibilities to optimize the EMFB implementation in dedicated hardware, using short coefficient word-lengths, sums of powers of two implementations for coefficients, and so forth.

Furthermore, it can be noted that due to the larger blocksize, OFDM requires significantly bigger data memory and coefficient storage in processor-based implementations.

One quite significant aspect is the needed baseband filtering in the receiver before the filter bank or FFT. The oversampled analysis bank acts as a high-quality channel selection filter, effectively suppressing adjacent channels and other interference components appearing in the range of the unused subcarriers. In the OFDM case, the attenuation capability 
of the DFT is rather limited, regarding the adjacent channels and other out-of-band interference sources that are not synchronized to the guard interval structure. Therefore additional highly selective digital baseband filtering is usually needed in OFDM, especially if the frequency domain guardbands between the adjacent frequency channels are to be minimized. Including the baseband filtering in the complexity comparison may change the measures significantly.

\section{CONCLUSION}

We have studied a new low-complexity per-subcarrier channel equalizer for FBMC transceiver for high-rate wideband communications over doubly-dispersive channel and analyzed its performance. It was shown that the coded errorrate performance of FBMC is somewhat better than that of the OFDM reference. It was also indicated that the performance of FBMC with a practical nonlinear power amplifier is similar to that of OFDM or even better. Further, FBMC is much less sensitive to frequency offsets than OFDM due to the possibility of using significantly lower number of subcarriers. The latter observation indicates also a potential for improved performance benefit in case of fast-fading channels. It was seen that an FBMC receiver can be operated without time synchronization prior to the receiver bank, also with higher-order modulations if AP-SCE is used. This leads to a simplified overall receiver structure.

The arithmetic complexity of FBMC is, no doubt, higher than that of the reference OFDM, but yet realistic with digital VLSI technologies. However, FBMC has various benefits over OFDM, like higher flexibility in choosing the design parameters. Especially, it can be emphasized that the receiver filter bank in FBMC acts as a high-quality frequency selective filter for all the signal components in the received band. This is in contrast to OFDM where transients are introduced to the signal components that are not synchronized to the guard interval structure, causing leakage of interference power also to noninterfered parts of the spectrum. Therefore, FBMC has the potential of providing sufficient attenuation for adjacent channels and other interferences, reducing the complexity of the baseband filtering of the receiver. On the other hand, this feature makes it feasible to have small frequency domain guard-bands between the asynchronous adjacent channel users, increasing the spectral efficiency. Selectivity provides also high robustness to narrowband interferences in the signal band, and a possibility to use multiple nonadjacent frequency slots for a single user in a flexible manner.

The numerical examples were mostly performed with high-end TMUX designs ( $K=5$ ) with relatively high stopband attenuation (about $50 \mathrm{~dB}$ ). However, results with lowercomplexity filter banks $(K=3$ ) (about $38 \mathrm{~dB}$ ) were also shown and even the case with $K=2$ (about $30 \mathrm{~dB}$ ) was tested. The conclusion is that even though the performance analysis assumed infinite attenuation for the subchannel stopbands, the performance degradation even with about $30 \mathrm{~dB}$ stopband attenuation is minor if the system does not need to suppress strong interfering signal components.
These results encourage further research on FBMC for beyond 3G communications. Such studies include development of robust synchronization and channel estimation techniques, as well as optimization of filter banks for low complexity with high flexibility. For example, efficient NPR filter bank designs form an interesting topic.

\section{APPENDIX}

\section{ICI AND ISI RELATED IMPULSE RESPONSES IN THE BASEBAND MODEL}

We first derive the equivalent baseband model for the lower ICI response using (1) and (5):

$$
\begin{aligned}
\tilde{v}_{k}[n]= & \left\{f_{k-1}[n] * \bar{h}_{k}[n]\right\} e^{-j(n k \pi / M)} \\
= & \left\{f_{k-1}[n] e^{-j(n k \pi / M)}\right\} *\left\{\bar{h}_{k}[n] e^{-j(n k \pi / M)}\right\} \\
= & \left\{f_{k-1}[n] e^{-j(n k \pi / M)} e^{-j((M+1) k \pi / 2 M)}\right\} \\
& *\left\{\bar{h}_{k}[n] e^{-j(n k \pi / M)} e^{+j((M+1) k \pi / 2 M)}\right\} \\
= & \left\{f_{k-1}[n] e^{-j(n+(M+1) / 2)(k \pi / M)}\right\} \\
& *\left\{\bar{h}_{k}[n] e^{j(-n+(M+1) / 2)(k \pi / M)}\right\} \\
= & f_{2 M-1}[n] * \bar{h}_{0}[n]=v_{0}[n] .
\end{aligned}
$$

Further,

$$
\begin{aligned}
v_{0}[n]= & f_{2 M-1}[n] * \bar{h}_{0}[n] \\
= & \left(-f_{0}^{c}[n]+j f_{0}^{s}[n]\right) *\left(\bar{h}_{0}^{c}[n]-j \bar{h}_{0}^{s}[n]\right) \\
= & -f_{0}^{c}[n] * \bar{h}_{0}^{c}[n]+f_{0}^{s}[n] * \bar{h}_{0}^{s}[n] \\
& +j\left(f_{0}^{s}[n] * \bar{h}_{0}^{c}[n]+f_{0}^{c}[n] * \bar{h}_{0}^{s}[n]\right) .
\end{aligned}
$$

Applying the relationships between the sine/cosine modulated analysis/synthesis filters that can be found in [26] and which apply for a PR TMUX,

$$
\begin{aligned}
& h_{k}^{s}[n]=(-1)^{k+K} f_{k}^{c}[n], \\
& f_{k}^{s}[n]=(-1)^{k+K} h_{k}^{c}[n],
\end{aligned}
$$

we finally obtain

$$
v_{0}[n]=j\left(f_{0}^{s}[n] * \bar{h}_{0}^{c}[n]+f_{0}^{c}[n] * \bar{h}_{0}^{s}[n]\right) .
$$

Although (A.2) and (A.4) use the noncausal form for the analysis filters, we can see that the introduced time delay does not affect (A.3) in any way. Further, since the delay is the same in both sine and cosine analysis filters, the real terms cancel each other out the same way they would do if causal expressions were used. Therefore, the lower ICI is purely imaginary in the baseband model. Likewise, we can write for the upper ICI response

$$
\begin{aligned}
\tilde{u}_{k}[n]= & \left\{f_{k+1}[n] * \bar{h}_{k}[n]\right\} e^{-j(n(k+1) \pi / M)} \\
= & \left\{f_{k+1}[n] e^{-j(n+(M+1) / 2)((k+1) \pi / M)}\right\} \\
& *\left\{\bar{h}_{k}[n] e^{j(-n+(M+1) / 2)((k+1) \pi / M)}\right\} \\
= & f_{0}[n] * \bar{h}_{2 M-1}[n]=u_{2 M-1}[n] .
\end{aligned}
$$


Now

$$
\begin{aligned}
u_{2 M-1}[n]= & \left(f_{0}^{c}[n]+j f_{0}^{s}[n]\right) *\left(-\bar{h}_{0}^{c}[n]-j \bar{h}_{0}^{s}[n]\right) \\
= & -f_{0}^{c}[n] * \bar{h}_{0}^{c}[n]+f_{0}^{s}[n] * \bar{h}_{0}^{s}[n] \\
& -j\left(f_{0}^{c}[n] * \bar{h}_{0}^{s}[n]+f_{0}^{s}[n] * \bar{h}_{0}^{c}[n]\right) \\
= & -j\left(f_{0}^{c}[n] * \bar{h}_{0}^{s}[n]+f_{0}^{s}[n] * \bar{h}_{0}^{c}[n]\right),
\end{aligned}
$$

so also the upper ICI response is purely imaginary.

For the impulse response of subchannel $k$, we can write in the baseband model

$$
\begin{aligned}
\tilde{t}_{k}[n]= & \left\{f_{k}[n] * \bar{h}_{k}[n]\right\} e^{-j(n k \pi / M)} \\
= & \left\{f_{k}[n] e^{-j(n+(M+1) / 2)(k \pi / M)}\right\} \\
& *\left\{\bar{h}_{k}[n] e^{+j(-n+(M+1) / 2)(k \pi / M)}\right\} \\
= & f_{0}[n] * \bar{h}_{0}[n]=t_{0}[n] .
\end{aligned}
$$

\section{ACKNOWLEDGMENTS}

The authors would like to thank the anonymous reviewers and the associate editor for their constructive comments and suggestions that helped to improve the manuscript, both in presentation and contents. This research was partly funded by Nokia.

\section{REFERENCES}

[1] R. van. Nee and R. Prasad, OFDM for Wireless Multimedia Communications, Artech House, Boston, Mass, USA, 2000.

[2] R. W. Chang, "Synthesis of band-limited orthogonal signals for multichannel data transmission," Bell System Technical Journal, vol. 45, pp. 1775-1796, 1966.

[3] B. R. Saltzberg, "Performance of an efficient parallel data transmission system," IEEE Transactions on Communications, vol. 15, no. 6, pp. 805-811, 1967.

[4] B. Hirosaki, "An analysis of automatic equalizers for orthogonally multiplexed QAM systems," IEEE Transactions on Communications, vol. 28, no. 1, pp. 73-83, 1980.

[5] B. Le Floch, M. Alard, and C. Berrou, "Coded orthogonal frequency division multiplex," Proceedings of the IEEE, vol. 83, no. 6, pp. 982-996, 1995.

[6] S. D. Sandberg and M. A. Tzannes, "Overlapped discrete multitone modulation for high speed copper wire communications," IEEE Journal on Selected Areas in Communications, vol. 13, no. 9, pp. 1571-1585, 1995.

[7] A. Vahlin and N. Holte, "Optimal finite duration pulses for OFDM," IEEE Transactions on Communications, vol. 44, no. 1, pp. 10-14, 1996.

[8] T. Wiegand and N. J. Fliege, "Equalizers for transmultiplexers in orthogonal multiple carrier data transmission," in Proceedings of the European Signal Processing Conference (EUSIPCO '96), vol. 2, pp. 1211-1214, Trieste, Italy, September 1996.

[9] S. Nedic, "An unified approach to equalization and echo cancellation in OQAM-based multi-carrier data transmission," in Proceedings of IEEE Global Telecommunications Conference (GLOBECOM '97), vol. 3, pp. 1519-1523, Phoenix, Ariz, USA, November 1997.

[10] L. Vandendorpe, L. Cuvelier, F. Deryck, J. Louveaux, and O. van de Wiel, "Fractionally spaced linear and decision-feedback detectors for transmultiplexers," IEEE Transactions on Signal Processing, vol. 46, no. 4, pp. 996-1011, 1998.

[11] K. Van Acker, G. Leus, M. Moonen, O. van de Wiel, and T. Pollet, "Per tone equalization for DMT-based systems," IEEE Transactions on Communications, vol. 49, no. 1, pp. 109-119, 2001.

[12] P. Siohan, C. Siclet, and N. Lacaille, "Analysis and design of OFDM/OQAM systems based on filterbank theory," IEEE Transactions on Signal Processing, vol. 50, no. 5, pp. 1170-1183, 2002.

[13] A. M. Wyglinski, P. Kabal, and F. Labeau, "Adaptive filterbank multicarrier wireless systems for indoor environments," in Proceedings of the 56th IEEE Vehicular Technology Conference (VTC '02), vol. 1, pp. 336-340, Vancouver BC, Canada, September 2002.

[14] B. Farhang-Boroujeny, "Multicarrier modulation with blind detection capability using cosine modulated filter banks," IEEE Transactions on Communications, vol. 51, no. 12, pp. 20572070, 2003.

[15] B. Farhang-Boroujeny and L. Lin, "Analysis of post-combiner equalizers in cosine-modulated filterbank-based transmultiplexer systems," IEEE Transactions on Signal Processing, vol. 51, no. 12, pp. 3249-3262, 2003.

[16] S.-M. Phoong, Y. Chang, and C.-Y. Chen, "DFT-modulated filterbank transceivers for multipath fading channels," IEEE Transactions on Signal Processing, vol. 53, no. 1, pp. 182-192, 2005.

[17] M. Vetterli, "Perfect transmultiplexers," in Proceedings of IEEE International Conference on Acoustics, Speech and Signal Processing (ICASSP '86), vol. 11, pp. 2567-2570, Tokyo, Japan, September 1986.

[18] H. S. Malvar, Signal Processing with Lapped Transforms, Artech House, Boston, Mass, USA, 1992.

[19] P. P. Vaidyanathan, Multirate Systems and Filter Banks, Prentice Hall, Englewood Cliffs, NJ, USA, 1993.

[20] T. Karp and N. J. Fliege, "Modified DFT filter banks with perfect reconstruction," IEEE Transactions on Circuits and Systems II: Analog and Digital Signal Processing, vol. 46, no. 11, pp. 1404-1414, 1999.

[21] J. Alhava and M. Renfors, "Adaptive sine-modulated/cosinemodulated filter bank equalizer for transmultiplexers," in Proceedings of the European Conference on Circuit Theory and Design (ECCTD '01), vol. 3, pp. 337-340, Espoo, Finland, August 2001.

[22] T. Ihalainen, T. Hidalgo Stitz, and M. Renfors, "On the performance of low-complexity ASCET-equalizer for a complex transmultiplexer in wireless mobile channel," in Proceedings of the 7th International OFDM-Workshop, Hamburg, Germany, September 2002.

[23] T. Ihalainen, T. Hidalgo Stitz, and M. Renfors, "Efficient percarrier channel equalizer for filter bank based multicarrier systems," in Proceedings of IEEE International Symposium on Circuits and Systems (ISCAS '05), pp. 3175-3178, Kobe, Japan, May 2005.

[24] T. Ihalainen, T. Hidalgo Stitz, and M. Renfors, "Performance comparison of LDPC-coded FBMC and CP-OFDM in beyond 3G context," in Proceedings of IEEE International Symposium 
on Circuits and Systems (ISCAS '06), pp. 2049-2052, Kos, Greece, May 2006.

[25] Y. Yuan, T. Ihalainen, M. Rinne, and M. Renfors, "Frequency domain equalization in single carrier transmission: filter bank approach," accepted to EURASIP Journal on Applied Signal Processing.

[26] A. Viholainen, J. Alhava, and M. Renfors, "Efficient implementation of $2 \mathrm{x}$ oversampled exponentially modulated filter banks," IEEE Transactions on Circuits and Systems II, vol. 53, pp. 1138-1142, 2006.

[27] J. Alhava and M. Renfors, "Complex lapped transforms and modulated filter banks," in Proceedings of the 2nd International Workshop on Spectral Methods and Multirate Signal Processing (SMMSP '02), pp. 87-94, Toulouse, France, September 2002.

[28] A. Viholainen, T. Hidalgo Stitz, J. Alhava, T. Ihalainen, and M. Renfors, "Complex modulated critically sampled filter banks based on cosine and sine modulation," in Proceedings of IEEE International Symposium on Circuits and Systems (ISCAS '02), vol. 1, pp. 833-836, Scottsdale, Ariz, USA, May 2002.

[29] A. Viholainen, J. Alhava, and M. Renfors, "Efficient implementation of complex modulated filter banks using cosine and sine modulated filter banks," Eurasip Journal on Applied Signal Processing, vol. 2006, Article ID 58564, 10 pages, 2006.

[30] E. A. Lee and D. G. Messerschmitt, Digital Communication, Kluwer Academic, Boston, Mass, USA, 2nd edition, 1994.

[31] J. G. Proakis, Digital Communications, McGraw-Hill, New York, NY, USA, 3rd edition, 1995.

[32] ITU-R, "Guidelines for evaluation of radio transmission technologies for IMT-2000,” Recommendation M.1225, 1997.

[33] T. B. Sorensen, P. E. Mogensen, and F. Frederiksen, "Extension of the ITU channel models for wideband (OFDM) systems," in Proceedings of the 62nd IEEE Vehicular Technology Conference (VTC '05), vol. 1, pp. 392-396, Dallas, Tex, USA, September 2005.

[34] T. I. Laakso, V. Vālimāki, M. Karjalainen, and U. K. Laine, "Splitting the unit: delay tools for fractional delay filter design," IEEE Signal Processing Magazine, vol. 13, no. 1, pp. 30 60, 1996.

[35] R. G. Gallager, "Low-density parity-check codes," IRE Transactions on Information Theory, vol. 8, pp. 21-28, 1962.

[36] C. Rapp, "Effects of HPA-nonlinearity on a 4-DPSK/OFDMsignal for a digital sound broadcasting system," in Proceedings of the 2nd European Conference on Satellite Communications, pp. 179-184, Liege, Belgium, October 1991.

[37] P. H. Moose, "Technique for orthogonal frequency division multiplexing frequency offset correction," IEEE Transactions on Communications, vol. 42, no. 10, pp. 2908-2914, 1994.

Tero Ihalainen received his M.S. degree in electrical engineering from Tampere University of Technology (TUT), Finland, in 2005. Currently, he is a Researcher and a Postgraduate student at the Institute of Communications Engineering at TUT, pursuing towards the doctoral degree. His main research interests are digital signal processing algorithms for multicarrier and frequency domain equalized single-carrier

modulation-based wireless communications, especially applications of multirate filter banks.

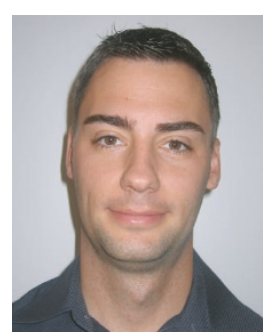

Tobias Hidalgo Stitz was born in 1974 in Eschwege, Germany. He obtained the M.S. degree in telecommunications engineering from the Polytechnic University of Madrid (UPM) in 2001, after writing his Masters Thesis at the Institute of Communications Engineering of the Tampere University of Technology (TUT). From 1999 to 2001, he was Research Assistant at TUT and is now working towards his doctoral degree there. His research interests include wireless communications based on multicarrier systems, especially focusing on filter bank based systems and other filter bank applications for signal processing.

Mika Rinne received his M.S. degree from TUT in signal processing and computer science, in 1989. He acts as Principal Scientist in the Radio Technologies Laboratory of Nokia Research Center. His background is in research of multiple-access methods, radio resource management, and implementation of packet decoders for radio communication systems. Currently, his interests are in research of protocols and algorithms for

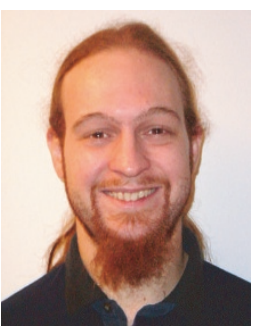
wireless communications including WCDMA, long-term evolution of $3 \mathrm{G}$ and beyond $3 \mathrm{G}$ systems.

Markku Renfors was born in Suoniemi, Finland, on January 21, 1953. He received the Diploma Engineer, Licentiate of Technology, and Doctor of Technology degrees from (TUT), Tampere, Finland, in 1978, 1981, and 1982, respectively. From 1976 to 1988 , he held various research and teaching positions at TUT. From 1988 to 1991, he was a Design Manager at the Nokia Research Center and Nokia Consumer Elec-

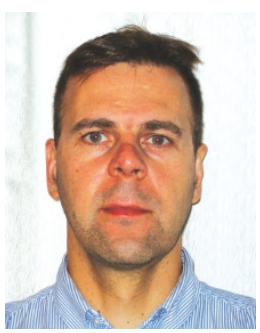
tronics, Tampere, Finland, where he focused on video signal processing. Since 1992, he has been a Professor and Head of the Institute of Communications Engineering at TUT. His main research areas are multicarrier systems and signal processing algorithms for flexible radio receivers and transmitters. 